\title{
THE
}

\section{The Effect of Breaking Waves on a Coupled Model of Wind and Ocean Surface Waves. Part II: Growing Seas}

Tobias Kukulka

Tetsu Hara

University of Rhode Island, thara@uri.edu

\section{Citation/Publisher Attribution}

Kukulka, T. and T. Hara, 2008: The Effect of Breaking Waves on a Coupled Model of Wind and Ocean Surface Waves. Part II: Growing Seas. J. Phys. Oceanogr., 38, 2164-2184, https://doi.org/10.1175/ 2008JP03962.1

Available at: https://doi.org/10.1175/2008JP03962.1

This Article is brought to you for free and open access by the Graduate School of Oceanography at DigitalCommons@URI. It has been accepted for inclusion in Graduate School of Oceanography Faculty Publications by an authorized administrator of DigitalCommons@URI. For more information, please contact digitalcommons-group@uri.edu. 


\title{
The Effect of Breaking Waves on a Coupled Model of Wind and Ocean Surface Waves. Part II: Growing Seas
}

\author{
Tobias Kukulka* and Tetsu Hara \\ Graduate School of Oceanography, University of Rhode Island, Narragansett, Rhode Island
}

(Manuscript received 4 December 2007, in final form 31 March 2008)

\begin{abstract}
This is the second part of a two-part investigation of a coupled wind and wave model that includes the enhanced form drag of breaking waves. The model is based on the wave energy balance and the conservation of air-side momentum and energy. In Part I, coupled nonlinear advance-delay differential equations were derived, which govern the wave height spectrum, the distribution of breaking waves, and vertical air side profiles of the turbulent stress and wind speed. Numeric solutions were determined for mature seas. Here, numeric solutions for a wide range of wind and wave conditions are obtained, including young, strongly forced wind waves. Furthermore, the "spatial sheltering effect" is introduced so that smaller waves in airflow separation regions of breaking longer waves cannot be forced by the wind. The solutions strongly depend on the wave height curvature spectrum at high wavenumbers (the "threshold saturation level"). As the threshold saturation level is reduced, the effect of breaking waves becomes stronger. For young strongly forced waves (laboratory conditions), breaking waves close to the spectral peak dominate the wind input and previous solutions of a model with only input to breaking waves are recovered. Model results of the normalized roughness length are generally consistent with previous laboratory and field measurements. For field conditions, the wind stress depends sensitively on the wave height spectrum. The spatial sheltering may modify the number of breaking shorter waves, in particular, for younger seas.
\end{abstract}

\section{Introduction}

Understanding coupled wind and wave dynamics plays a key role in predicting transfer processes through the air-sea interface (Melville 1996; Jähne and Haußecker 1998). Above the air-sea interface, surface waves perturb the airflow, which causes a "wave form drag" so that energy and momentum exchange between water and air takes place (Phillips 1977; Belcher and Hunt 1998). In most previous coupled wind and wave models, only the form drag of nonbreaking waves has been considered, as reviewed by Komen et al. (1996) and Cavaleri (2006). Airflow separation of breaking waves, however, leads to enhanced momentum fluxes to the wave field (Banner 1990; Kudryavtsev and Makin 2001; Makin and Kudryavtsev 2002). This paper

* Current affiliation: Woods Hole Oceanographic Institution, Woods Hole, Massachusetts.

Corresponding author address: Tobias Kukulka, Graduate School of Oceanography, University of Rhode Island, Narragansett, RI 02882.

E-mail:kukulka@whoi.edu is the second part of a two-part investigation that explores how a random distribution of breaking waves influences coupled wave and wind dynamics.

Previously Kukulka and Hara (2008, hereafter referred to as Part I) developed a coupled wind and wave model that includes the enhanced form drag of breaking waves. The model is expressed in terms of coupled nonlinear advance-delay differential equations, governing the wave height spectrum, the distribution of breaking waves, and vertical air-side profiles of the turbulent stress and the wind speed. Furthermore, numerical solutions for fully developed seas were obtained. In this study, we investigate the solutions for a wide range of wind and wave conditions, including young growing wave fields.

For practical air-sea flux parameterizations, it is particularly interesting to understand the systematic dependence of our model on environmental conditions, such as wind speed and wave age. The wave age is here defined by the ratio of the phase speed at the spectral peak, $c_{p}$, to the total air friction velocity, $u_{*}=\sqrt{\tau_{0} / \rho_{a}}$ ( $\tau_{0}$ and $\rho_{a}$ symbolize the total wind surface stress and the density of air, respectively). 
For moderate wind conditions, observations of winddriven seas indicate that the gravity wave height spectrum can be related to the wave age [see, e.g., discussion by Donelan in Komen et al. (1996)]. For a given peak wavenumber $k_{p}$ and different wind conditions, the wave height spectrum increases with wave age for waves at wavenumbers $k$ that are relatively close to the spectral peak (say, for $2 k_{p}<k<10 k_{p}$ ).

The behavior of the gravity short-wave spectrum (say wavelengths between 0.05 and $2.0 \mathrm{~m}$ ) is less understood; some field measurements indicate that the spectrum is independent of wave age and wind speed (Banner et al. 1989; Melville and Matusov 2002), while other laboratory observations show a spectral increase with wind speed (Jähne and Riemer 1990). For high wind conditions, as they occur under tropical cyclones, the behavior of the wave height spectrum is poorly understood.

Laboratory and field measurements also suggest a systematic dependence of the total air-sea momentum flux (wind stress) on wind speed and wave age, which is consistent with the fact that momentum is fluxed to the wave field (Jones and Toba 2001). Often, the wind stress is parameterized by the drag coefficient $c_{D}=$ $u_{*}^{2} / U_{10}^{2}$, where $U_{10}$ symbolizes the reference wind speed at $10-\mathrm{m}$ height. For neutrally stable conditions, it is convenient to express the drag coefficient in terms of aerodynamical roughness length

$$
z_{0}=h_{\text {ref }} \exp \left(-\frac{\kappa U_{10}}{u_{*}}\right),
$$

or the normalized roughness length $r=z_{0} g u_{*}^{-2}$ (Charnock coefficient) with the acceleration of gravity $g$, the von Kármán constant $\kappa=0.4$, and the reference height $h_{\text {erf }}=10 \mathrm{~m}$. Observations of the Charnock coefficient, to date, span a wide range with approximately three orders of magnitude. This reflects, on one hand, the enormous challenge of accurately determining the wind stress and, on the other, poor understanding of the Charnock coefficient dependency on environmental conditions. In spite of the scatter in the data, recent investigations of carefully selected field observations (intermediate wave ages to fully developed seas) indicate a decrease in the Charnock coefficient with wave age for moderate wind speeds (say, $U_{10}=7-25 \mathrm{~m} \mathrm{~s}^{-1}$ ) (Drennan et al. 2003; Hwang 2005; Smedman et al. 2003). For very young wave fields, as observed in the laboratory, the Charnock coefficient increases with wave age (Toba et al. 1990). Under very high wind speed conditions ( $\mathrm{say},>30 \mathrm{~m} \mathrm{~s}^{-1}$ ), recent observations suggest that the drag coefficient levels off for laboratory wave fields (Donelan et al. 2004) and under tropical cyclones (Powell et al. 2003; Jarosz et al. 2007).
The breaking wave effect on the drag coefficient was investigated by Kudryavtsev and Makin (2001) and Makin and Kudryavtsev (2002). Their results indicate that breaking waves contribute significantly to the total wind stress for wind speeds greater than $U_{10}=10 \mathrm{~m} \mathrm{~s}^{-1}$ and that the influence of breaking waves increases with wind speed and inverse wave age (younger seas).

The following aspects of our model follow closely the approach from Kudryavtsev and Makin (2001): 1) partitioning of the wave-induced stress into two parts due to breaking and nonbreaking waves, 2) parameterization of the breaking wave stress, and 3) conservation of the total air-sea momentum flux at the sea surface. In addition, we impose momentum and energy conservation at each height of the wave boundary layer (the thin layer above the air-sea interface that is influenced by wave-induced fluxes). Therefore, we vertically resolve the wave boundary layer. Unlike the approach from Makin and Kudryavtsev, we consider here the energy input to breaking waves in the wave energy balance. The breaking-wave energy input is particularly important for young, strongly forced wave fields.

To understand the greatest possible influence of breaking waves, we developed previously a coupled wind and wave model based on wave energy balance and air-side energy and momentum conservation (Kukulka et al. 2007, hereafter KHB). Such a model may be applicable to strongly forced wind waves observed in the laboratory. For field conditions, however, the input to nonbreaking waves cannot be neglected.

The goal of this study is to develop a coupled wind and wave model that includes the effect of breaking waves and is applicable to the full wave age range from mature ocean seas to very young strongly forced laboratory waves. In the next section we will briefly review major model assumptions (section 2), which is followed by a discussion on model results (section 3 ). In section 4 we will examine how the "spatial sheltering effect" modifies the results from section 3. The paper is concluded with the discussion of section 5 .

\section{Review of theory}

We will first review briefly the system of governing equations derived in Part I. Later, in section 4, we will introduce the spatial sheltering effect and rederive the equations in more detail.

\section{a. Coupled wind-wave model with input to breaking waves}

Based on the conservation of wave energy and the conservation of air-side energy and momentum, in Part I we showed that the wave height curvature spectrum $B$ [here $B(k, \theta)=k^{4} \Psi(k, \theta)$, where $\Psi(k, \theta)$ is the two- 
dimensional wavenumber spectrum, $k$ is the angular wavenumber measured in SI units, and $\theta$ is the angle of propagation relative to the wind], the distribution of breaking waves $\Lambda[\Lambda(k, \theta) k d \theta d k$ is the average breaking crest length per unit surface area (Phillips 1985)], the mean wind speed $u$, and the turbulent wind stress $\tau_{t}$ is governed by the following system of nondimensionalized equations:

$$
\begin{aligned}
\frac{d S}{d K}= & S-M_{b}-M_{w}, \\
\frac{d U}{d K}= & \frac{1}{2} U-\left(S+S_{w}\right)^{-1}\left(E_{b}+\delta_{w}^{-1 / 2} E_{w}-U M_{b}\right. \\
& \left.+\kappa^{-1} S^{3 / 2}\right),
\end{aligned}
$$

which need to be solved for the normalized turbulent stress $S=\tau_{t} /\left(\rho_{a} c_{2}\right)$ and normalized wind speed $U=u / c$. Here $\tau_{t}(k)$ is the turbulent stress evaluated at $z=\epsilon / k, k$ is the wavenumber, $\epsilon$ is the slope of breaking waves, $\rho_{a}$ is air density, $c$ is the wave phase speed, and $u$ is the mean wind speed evaluated at $z=\epsilon / k$. The normalized wavenumber $K$ is related to the true wavenumber $k$ such that $d k=d k / k$. The constant coefficient $\delta_{\epsilon}$ is defined by $\delta_{\epsilon} \equiv \delta / \epsilon$ and $\delta$ is the normalized inner layer height. The normalized nonbreaking wave stress $S_{w}$ is calculated as

$$
\frac{d S_{w}}{d K}=S_{w}+M_{w}
$$

The normalized flux terms in the governing nondimensional equations are

$$
\begin{aligned}
M_{b} & =\int_{-\pi / 2}^{\pi / 2} s_{p}\left[k \beta_{\Lambda} \Lambda(K, \theta)\right](U \cos \theta-1)^{2} \cos \theta d \theta \\
M_{w} & =S \int_{-\pi / 2}^{\pi / 2} s_{\beta} c_{\beta} B(K-\Delta, \theta) h_{\beta}(\theta) \cos \theta d \theta \\
E_{b} & =\int_{-\pi / 2}^{\pi / 2} s_{p}\left[k \beta_{\Lambda} \Lambda(\mathrm{K}, \theta)\right][U \cos \theta-1]^{2} d \theta \\
E_{w} & =S \int_{-\pi / 2}^{\pi / 2} s_{\beta} c_{\beta} B(K-\Delta, \theta) h_{\beta}(\theta) d \theta
\end{aligned}
$$

where $\theta$ denotes the angle between the directions of wind and wave propagation, $h_{\beta}=\cos ^{2} \theta$ is the directionality of the wave growth rate, and the factors $s_{p}, s_{\beta}$ equal one for forced waves and zero otherwise. The constant delay or advance coefficient $\Delta$ is defined by $\Delta \equiv-\log (\delta / \epsilon)$. The wave height curvature spectrum (or saturation spectrum) $B$ and the breaking wave distribution $\Lambda$ are given by

$$
\begin{aligned}
c_{\beta} B(K, \theta) & =\left[\delta_{\epsilon} S(K+\Delta) \frac{h_{\beta}(\theta)}{\mu^{-2}-\gamma^{2} \mu_{\Lambda}^{-2} s_{p}(U \cos \theta-1)^{2}}\right]^{1 / 2}, \\
k \beta_{\Lambda} \Lambda(K, \theta) & =\gamma^{2} \mu_{\Lambda}^{-2}\left[c_{\beta} B(K, \theta)\right]^{3},
\end{aligned}
$$

for $B(K, \theta)<B_{\text {sat }}$ and $s_{\beta}=1$, and for $B(K, \theta) \rightarrow B_{\text {sat }}$ by

$$
\begin{aligned}
c_{\beta} B(K, \theta) & =c_{\beta} B_{\text {sat }}, \\
k \beta_{\Lambda} \Lambda(K, \theta) & =\frac{\gamma^{2} \delta_{\epsilon} h_{\beta}(\theta) s_{\beta} S(K+\Delta)+\gamma^{2}\left(\mu_{\Lambda}^{-2}-\mu^{-2}\right)\left(c_{\beta} B_{\text {sat }}\right)^{2}}{1-\gamma^{2} s_{p}[U \cos \theta-1]^{2}} c_{\beta} B_{\text {sat }} .
\end{aligned}
$$

Here $c_{\beta}$ is the coefficient of the wave growth rate, $\beta_{\Lambda}=$ $2 c_{\gamma} \epsilon, c_{\gamma}$ is the drag coefficient of breaking wave crests, $\gamma^{2}=\beta_{\Lambda} / b^{\prime}, b^{\prime}=b \rho_{w} / \rho_{a}, b$ is the coefficient of the breaking energy loss, and $\rho_{w}$ is water density. The coefficients $\mu$ and $\mu_{\Lambda}$ are related to the proportionality constant of the dissipation and nonlinear interaction terms in the wave energy equation. In this study, we set $\mu=\mu_{\Lambda}$, so that nonlinear interactions are neglected (see discussion in Part I). The model Eqs. (1) to (11) have been solved in Part I for mature seas. If our model is applied to growing wave fields, there are more uncertainties in the model coefficients, and the boundary conditions change, as discussed in the following subsection.

\section{b. Boundary conditions and model coefficients}

\section{1) Model coefficients}

As discussed in Part I, it is essential to constrain six critical model parameters: two height input coefficients: $\delta$ and $\epsilon$, two wave input coefficients: $c_{\beta}$ and $\gamma$, as well as the two wave height spectrum coefficients: $\mu$ and $B_{\text {sat }}$. The coefficients $\delta, \epsilon$ and the input coefficient to nonbreaking waves $c_{\beta}$ are held constant as in Part I; that is, $\delta=0.05, \epsilon=0.3$, and $c_{\beta}=25$. The input coefficient to breaking waves, $\gamma$, is by default set to 0.07 as in Part I. To investigate, however, the extent of the possible breaking wave effect, we will examine solutions for the full range of breaking wave input parameter $\gamma$ with 
$0.04 \leq \gamma \leq 0.5$ (KHB), corresponding to the uncertainty of the breaking energy dissipation coefficient $b$; that is, $0.003<b<0.07$ ).

In Part I we identified two parameters, $B_{\text {sat }}$ and $\mu$, that determine the wave height spectrum for high and low wavenumbers, respectively. For mature seas, $0.125<\mu<0.813$ and $0.001<B_{\text {sat }}<0.005$ : typical estimates are given by $B_{\text {sat }}=0.002$ and $\mu=0.6$. Observations suggest that the saturation level $B_{\text {sat }}$, however, changes with wind speed and wave age (Komen et al. 1996). In particular, there is evidence that $B_{\text {sat }}$ increases for growing seas. Therefore, in this study we set somewhat arbitrary but practical limits of $B_{\text {sat }}$ to $B_{\text {sat }} \rightarrow$ $\infty$ as the upper limit and the lower limit to $B_{\text {sat }}=5 \times$ $10^{-4}$. Note that, even though $B_{\text {sat }} \rightarrow \infty, B$ is still bounded by

$$
B(k, \theta) \rightarrow \frac{8}{3 \pi} \frac{1}{c_{\beta}}
$$

for high $k$, as discussed in the appendix.

Besides the model coefficients, boundary conditions critically determine the solutions.

\section{2) Boundary CONDitions}

Since the governing equations are third order, three boundary conditions for the dependent variables $S, U$, and $S_{w}$ are needed. As in Part I, we set $u$ asymptotically to zero at the sea surface. For numeric solutions, $u$ is set to zero at finite high $k_{1}$ so that the solutions presented here are independent of the actual value of $k_{1}$. The boundary value of the other two variables $S$ and $S_{w}$ are given at $k=k_{0}$, which is the lower wavenumber limit of the model calculation. The subscript " 0 " denotes a variable evaluated at $k=k_{0}$. Using this notation, $S_{0}=$ $u_{* 0}^{2} / c_{0}^{2}$ is related to a wave-age-like parameter $c_{0} / u_{* 0}$, which we refer to as "boundary wave age."

Strictly speaking, our coupled model is applicable only in the equilibrium range of the surface wave spectrum, where the nonlinear wave interaction is either negligible or simply proportional to the wind input and dissipation. Therefore, a wave model with a more accurate nonlinear interaction term [such as WAM (Komen et al. 1996) or WAVEWATCH (Tolman 1999)] should be used to predict the wave spectrum near the peak, and our model can be used to provide the tail (equilibrium range) of the spectrum. Then, $k=k_{0}$ is interpreted as the lower bound of the equilibrium range, which can be very different from the peak wavenumber. Hence, the boundary value $S_{0}$ may not be related to the true wave age, and $S_{w 0}$ may be greater than zero.

Alternatively, we may simply extend our coupled model to the spectral peak of a growing wave field and set $B=\Lambda=0$ for $k<k_{0}$. (We will call this an "idealized growing sea model.") In this approach we anticipate that the boundary wave age $\mathrm{c}_{0} / u_{* 0}$ is closely related to the real wave age $c_{p} / u_{*}$, if $k_{0}$ is close to the true spectral peak and the wind input to waves beyond the peak is relatively weak (here $c_{p}$ is the peak phase speed and $u_{*}$ the total air friction velocity). Since the nonlinear interaction is known to be significant near the spectral peak, this approach will not accurately predict the wave spectrum and breaking statistics near the peak. Nevertheless, the model may provide reasonable estimates of overall energy/momentum balance (i.e., energy/momentum balance integrated over the entire wavenumber range) and the drag coefficient, as the nonlinear interaction redistributes-but not generates or dissipates-energy and momentum. The same approach was taken by KHB to model very young seas with input to breaking waves only.

Since $S_{0}$ is related to the state of the sea surface in either approach, we will investigate below how solutions depend on $S_{0}$. The other boundary condition $S_{w 0}$ is related to the momentum input to nonbreaking waves with $k<k_{0}$. For given $S_{0}, S_{w 0}$ is set to zero below, consistent with the idealized growing sea model. The dependence of solutions on $S_{w 0} \neq 0$ for a given $S_{0}$ can be investigated by shifting (in $k$ ) solutions with $S_{w 0}=0$ and different values of $S_{0}$ until $S$ coincides at $k_{0}$.

To integrate the system from $K=K_{0}$ to $K_{1}$, one must specify $M_{w}$ and $E_{w}$ between $K_{0}$ and $K_{0}+\Delta$, which requires knowledge of $U$ between $K_{0}-\Delta$ and $K_{0}$. In the calculations below we set $M_{w}=E_{w}=0$ between $K_{0}$ and $K_{0}+\Delta$. This is consistent with the assumption that $B=\Lambda=0$ for $k<k_{0}$ in the idealized growing sea model.

\section{Results}

Here, we will present the solutions of the idealized growing seas for a wide range of $S_{0}$ (i.e., for a wide range of wave age). As discussed earlier, these solutions (or part of them) may be interpreted as equilibrium range model results, which are attached to more complex wave fields around their peaks.

\section{a. Solutions depend sensitively on $B_{\text {sat }}$}

First, the solutions of $S, U, B$, and $\Lambda$ for $\gamma=0.07$ and $\mu=0.6$ (default values) and different $B_{\text {sat }}$ are shown in Figs. 1-4. We have found that the solutions for growing seas are generally insensitive to the value of $\mu$ : $\mu$ is only important for $B<B_{\text {sat }}$ close to peak, where the effect of breaking waves is negligible. Therefore, the effect of varying $\mu$ is not discussed here. 

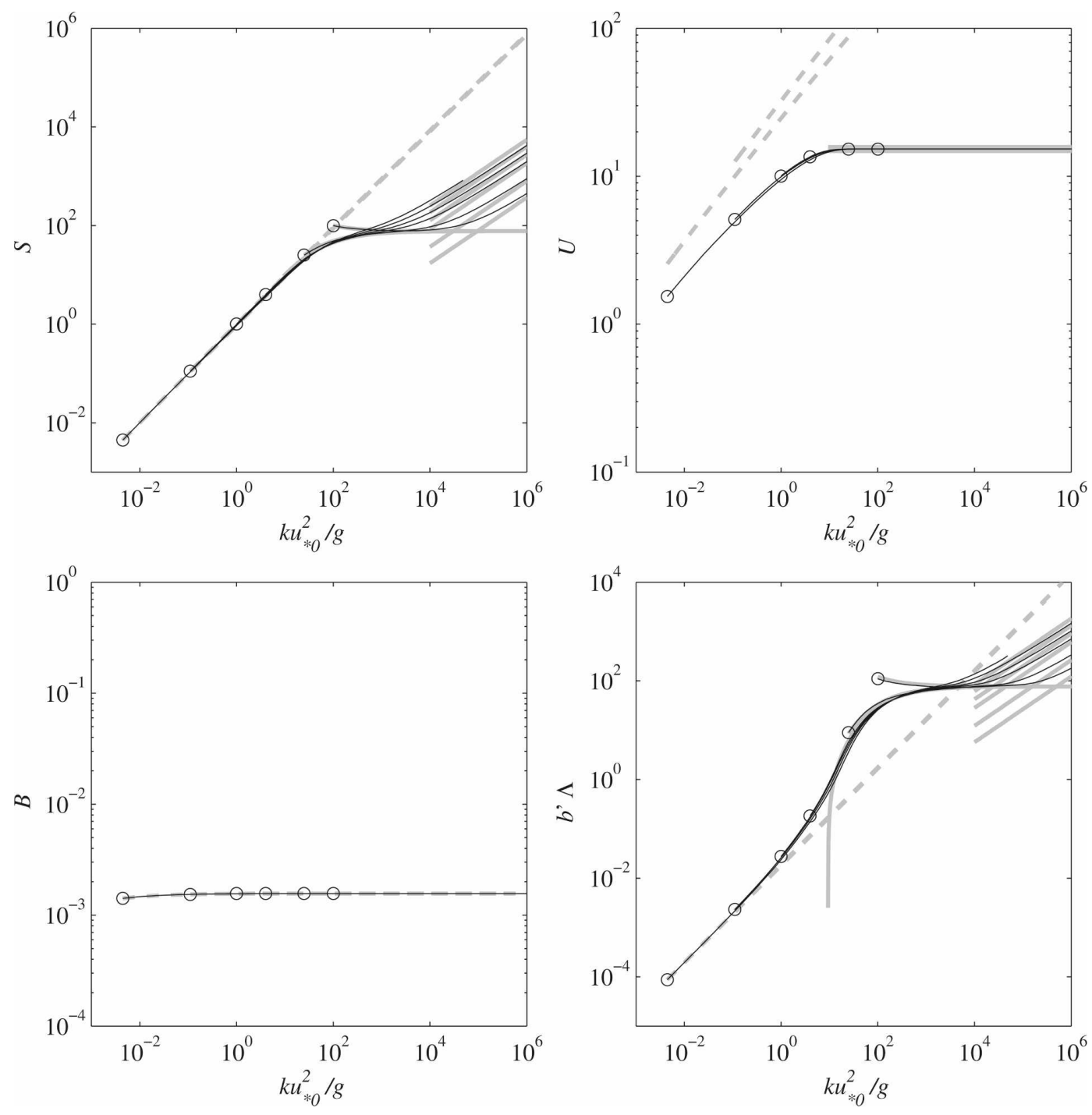

FIG. 1. Numeric solutions (black lines) for $B_{\text {sat }}=5 \times 10^{-4}, \gamma=0.07$, and boundary wave ages 15, 3.0, 1.0, 0.5, 0.2 , and 0.1 (corresponding to $S_{0}=0.0044,0.11,1.0,4.0,25,100$, indicated by circles from left to right). Solution without input to breaking waves (gray dashed line, $S_{0}=0.0044$ ), solution with only input to breaking waves (gray solid line, $S_{0}=S_{\min }, 25,100$ ), and asymptotic solutions (A28) for different $\tau_{w}$ (parallel straight gray lines).

The wavenumber is normalized in terms of the boundary friction velocity $u_{* 0}$ (which is expected to be close to the true friction velocity for the idealized growing sea) so that the solutions with decreasing $S_{0}$ show the development of the wind and wave field with wave age for fixed $u_{* 0}$ and $B_{\text {sat }}$.

The normalized turbulent stress $S$ and normalized wind speed $U$ reveal important information about the coupled wind-wave system. First, notice that the circles in the top left panel, corresponding to a particular $S$ boundary condition, are on top of the constant stress line $S=\tau_{0} k /\left(\rho_{\alpha} g\right)$. At the wavenumber where $S$ deviates from the constant stress line, the turbulent stress is significantly reduced due to momentum fluxes into waves. This wavenumber can be compared to the "sheltering wavenumber" from Hara and Belcher (2002). The $U$ graph (top right), on the other hand, concisely shows if breaking waves dominate the wind input, as in this case $U$ converges to the constant found in KHB. Note that for $U<1$ breaking waves are not forced by the wind.

For the lowest value of $B_{\text {sat }}=0.0005, B$ converges quickly to $B_{\text {sat }}$ (bottom left, Fig. 1). For the two lower $S_{0}$, corresponding to more developed seas, the distribution of breaking waves at low normalized wavenumbers coincides with the nonbreaking (NB) solution (gray dashed line, see the appendix), indicating the dominance of wind input to nonbreaking waves (bottom 

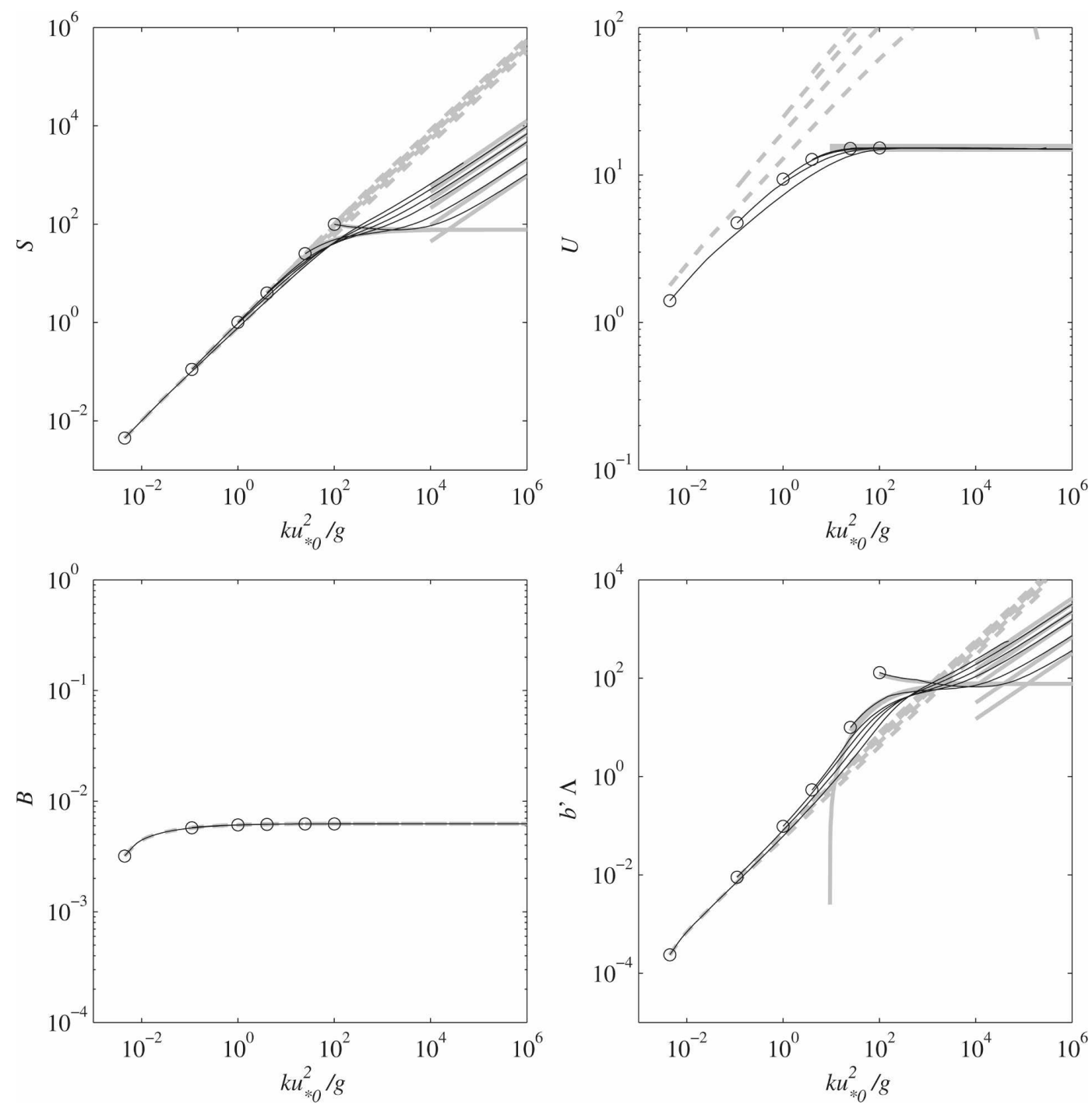

FIG. 2. As in Fig. 1, but for $B_{\text {sat }}=0.002$.

right, Fig. 1). The momentum uptake of these longer waves is relatively small since the total stress is approximately constant (top left, Fig. 1). As the normalized wavenumber increases, solutions first converge to the breaking-only (BO) solution of KHB (gray solid line, see the appendix) and then to the breaking-dominant (BD) solution (parallel straight gray lines, see the appendix). Therefore, the breaking wave effect is more pronounced for shorter, strongly forced (young) wind waves, which was suggested by KHB. The transition from the BO solution to the BD solution is discussed in the appendix. Note in particular that $\Lambda$ at large $k$ is asymptotically constant in the BO solution and is proportional to $k^{3 / 2}$ in the $\mathrm{BD}$ solution.

These results highlight at least two important wavenumber scales: first, the wavenumber at which $B$ tran- sitions to $B_{\text {sat }}$ and, second, a wavenumber at which the wind input changes from a nonbreaking- to a breakingwave-dominated input. The latter wavenumber roughly coincides with the wavenumber at which the turbulent stress is significantly reduced, as the input to nonbreaking is relatively small in this case.

When $B_{\text {sat }}$ is increased to 0.002 (Fig. 2), the solutions remain qualitatively similar, but $B$ and $b^{\prime} \Lambda$ at low normalized wavenumbers are raised. Note that for the NB solution and low $B_{\text {sat }}, \Lambda$ varies approximately linearly in $B_{\text {sat }}$, as suggested by (51) (see discussion in the appendix). Intuitively, an elevated wave spectrum increases the wind input to nonbreaking waves, which must be balanced by an enhanced wave dissipation, that is, an enhanced $\Lambda$.

The behavior of the solutions becomes more complex 

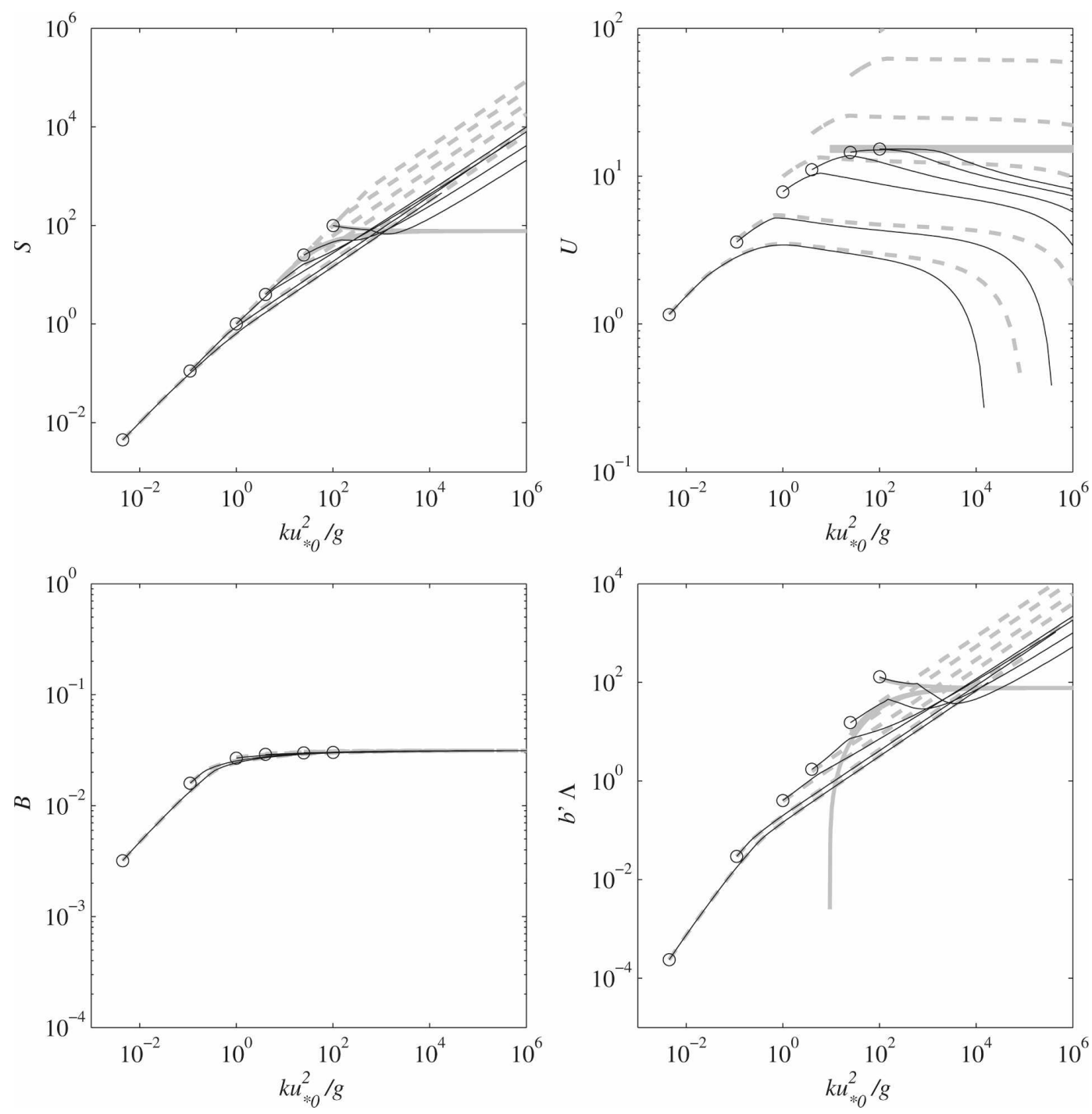

FIG. 3. Numeric solutions (black lines) for $B_{\mathrm{sat}}=0.01, \gamma=0.07$, and boundary wave ages $15,3.0,1.0,0.5,0.2$, and 0.1 (corresponding to $S_{0}=0.0044,0.11,1.0,4.0,25,100$, indicated by circles from left to right). Solution without input to breaking waves (gray dashed line); solution with only input to breaking waves (gray solid line), $S_{0}=S_{\min }$, 25,100 .

if the total input to nonbreaking waves is significant and input to both breaking and nonbreaking waves at a single wavenumber is important (Fig. 3). For $B_{\text {sat }}=$ 0.01 (a value close to its limit of $8 /\left(3 \pi c_{\beta}\right) \approx 0.034$; see the appendix), the input to nonbreaking waves dominates at low wavenumbers, as one expects from the previously discussed low $B_{\text {sat }}$ cases. Because $B_{\text {sat }}$ is elevated, more momentum is fluxed to waves, so the spectral sheltering is stronger. For the two lower $S_{0}$, corresponding to more developed seas, breaking waves play a relatively small role so that the solutions are well approximated by the NB solution for the entire wavenumber range. For greater $S_{0}$ (intermediate and younger wave ages), however, the wind speed profiles indicate that breaking waves contribute significantly to the wave energy input closer to the spectral peak. For very young seas, the model approaches the $\mathrm{BO}$ solution close to the spectral peak. Unlike the low $B_{\text {sat }}$ case, Fig. 3 indicates that the solutions for younger seas are sensitive to the advance-delay effect of the governing equations. This is because the input to nonbreaking waves near the peak is significant. The solutions tend to behave differently above and below $k=k_{0} \epsilon / \delta$ because we have set $B=0$ for $k<k_{0}$; that is, $B$ is discontinuous at $k=k_{0}$. If $B$ decays more gradually below $k_{0}$, this discontinuity will disappear.

The picture becomes somewhat simpler without liming $B_{\text {sat }}$ (Fig. 4). Here, the input to breaking waves is 

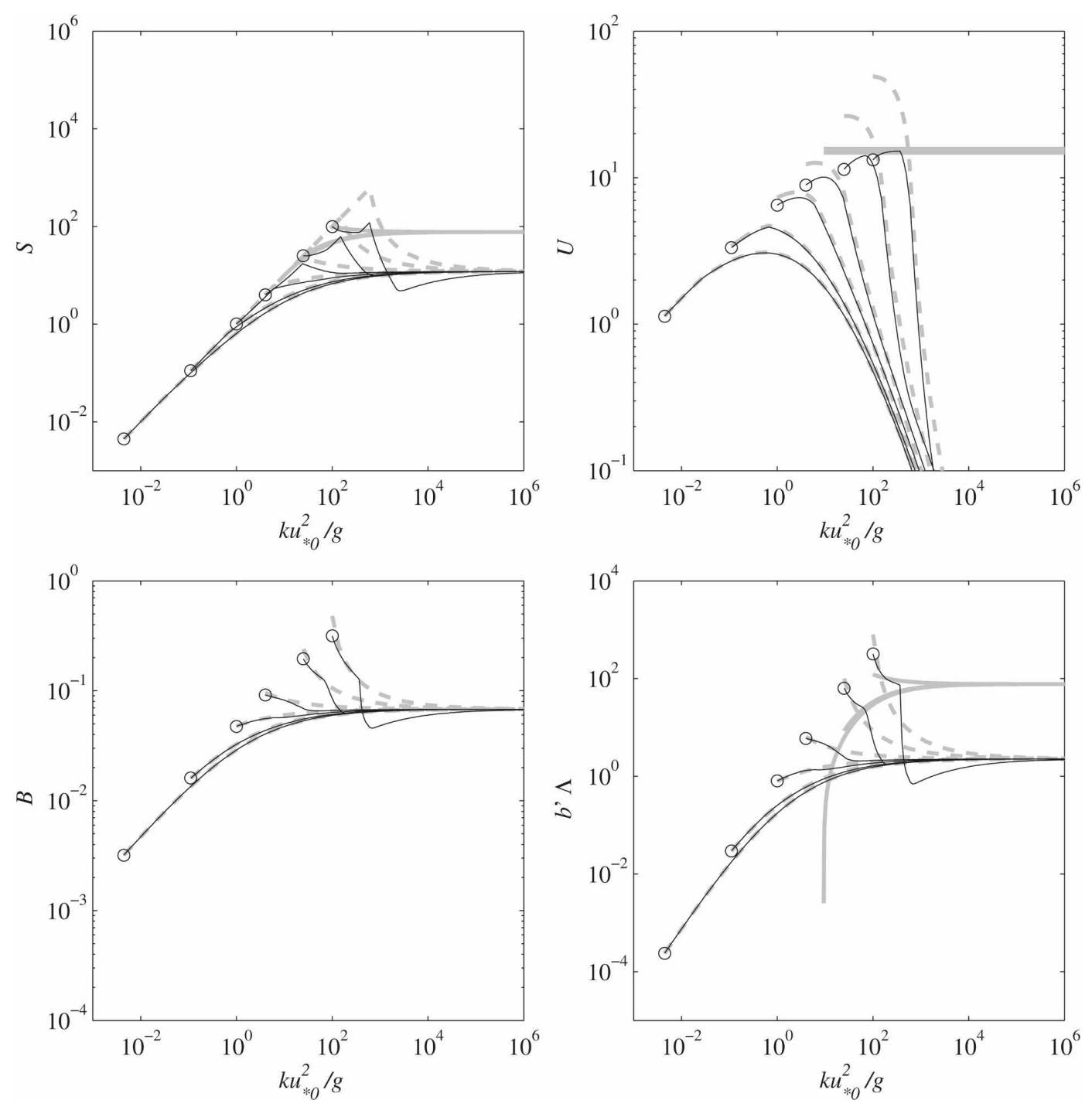

FIG. 4. As in Fig. 3, but without limiting $B_{\text {sat }}$.

relatively weak so that the solution is approximately described by the NB solution. The effect of breaking waves is most pronounced for younger wave fields where breaking waves can introduce local extreme points as well as sign changes in the curvature of $B$. In the next subsection, we will investigate the sensitivity of our results for enhanced breaking wave input parameter $\gamma$.

\section{b. Breaking parameter $\gamma$ also influences the solution}

In Part I, we determined the default value of the input coefficient to breaking waves to $\gamma=0.07$ so that the total air-sea momentum flux is consistent with observations for mature seas. The specified value is close to the lower limit $\gamma=0.04$ found in KHB. Considering the large uncertainties in the breaking-related parameters, we will also discuss solutions for the upper limit $\gamma=0.5$.

Figures 2 and 5 contrast the results at $\gamma=0.07$ and 0.5 with the same $B_{\text {sat }}=0.002$. The top left panels show that the enhanced input to breaking waves causes an increased momentum and energy uptake by breaking waves and, therefore, a larger spectral sheltering effect. Note also that the increasing $\gamma$ reduces the high $k$ magnitude of $\Lambda$, as found previously in KHB. Therefore, the wind input to breaking waves is enhanced but the number of breaking waves is reduced. The enhanced input coefficient to breaking waves also increases the total air-sea momentum flux, which we will discuss in the next subsection. 

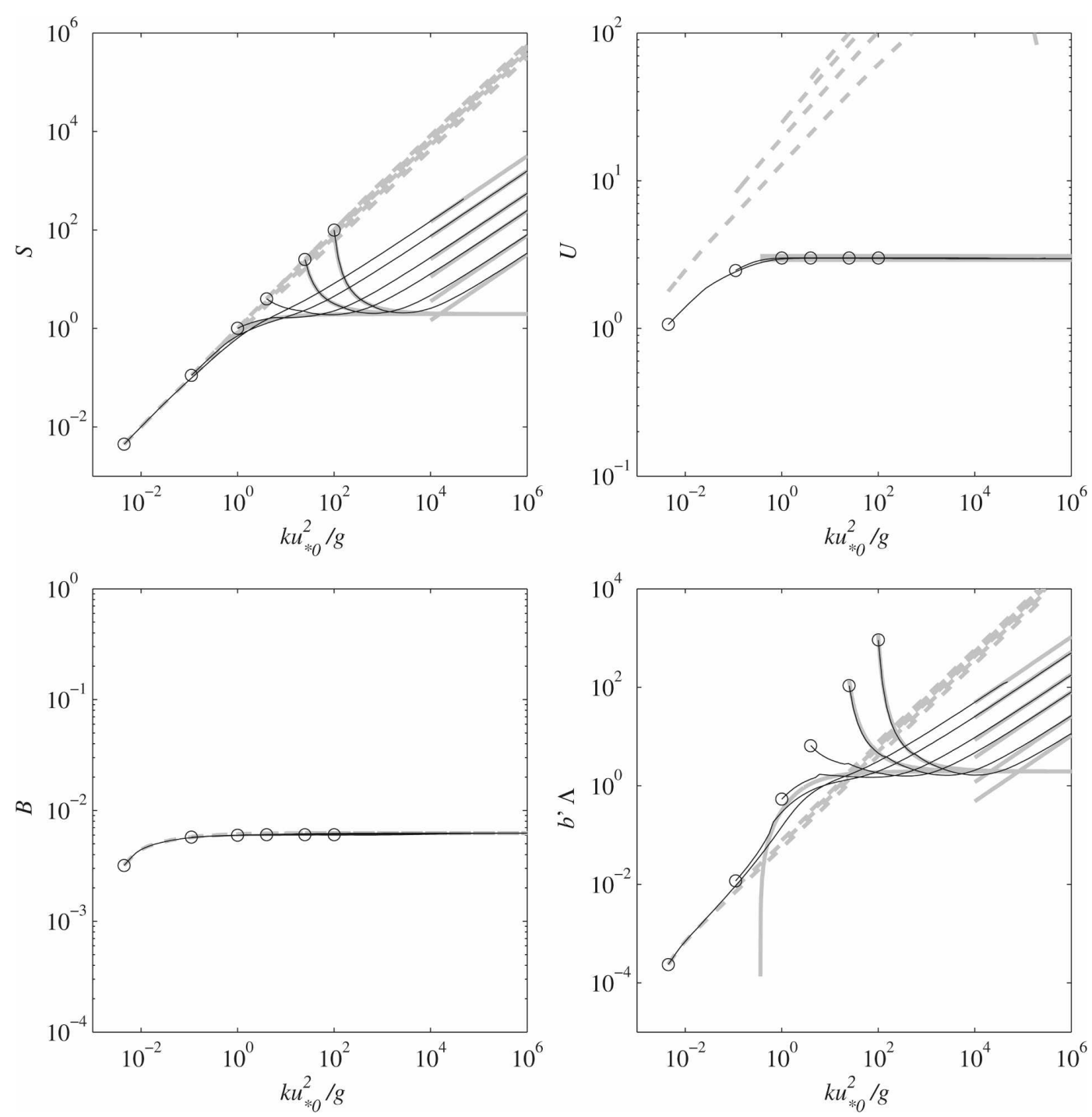

FIG. 5. Numeric solutions (black lines) for $B_{\text {sat }}=0.002, \gamma=0.5$, and boundary wave ages $15,3.0,1.0,0.5,0.2$, and 0.1 (corresponding to $S_{0}=0.0044,0.11,1.0,4.0,25,100$, indicated by circles from left to right). Solution without input to breaking waves (gray dashed line, $S_{0}=0.0044$ ), solution with only input to breaking waves (gray solid line, $S_{0}=S_{\text {min }}, 25,100$ ), and asymptotic solutions (A28) for different $\tau_{w}$ (parallel straight gray lines).

\section{c. Momentum flux}

In this section we will calculate the total momentum fluxed into the idealized growing seas and express it in terms of normalized roughness length (or Charnock coefficient), $r=z_{0} g / u_{*}^{2}$, where $z_{0}$ is the aerodynamical roughness length (see also Part I). The roughness length $z_{0}$ is defined through the logarithmic wind speed profile $u(z)=u_{*} / \kappa \ln \left(z / z_{0}\right)$ for a neutrally stable atmosphere. Here, we set $u_{*}=u_{* 0}$ so that the input to waves with $k<k_{0}$ equals zero. The Charnock coefficient is presented for different values of $\gamma$ and as a function of boundary wave age, $c_{0} / u_{* 0}=S_{0}^{-1 / 2}$, which is a proxy for the real wave age (Fig. 6). Upper and lower limits are estimated for each $\gamma$ based on the uncertainties of the wave height spectrum, that is, uncertainties in the parameters $\mu$ and $B_{\text {sat }}$.

As expected, the Charnock coefficient increases for increasing $\gamma$, as the input to breaking waves increases also. Furthermore, the range of solutions becomes narrower for enhanced $\gamma$ because the relative input to nonbreaking waves decreases; that is, the influence of the wave height spectrum declines. With the lowest value of $\gamma=0.04$ the momentum flux to nonbreaking waves is significant and the Charnock coefficient strongly depends on the spectral level. For the largest $B_{\text {sat }}$ and $\mu$, the solution converges to the upper bound of the NB model (dashed gray line in Fig. 6) as the wave age 


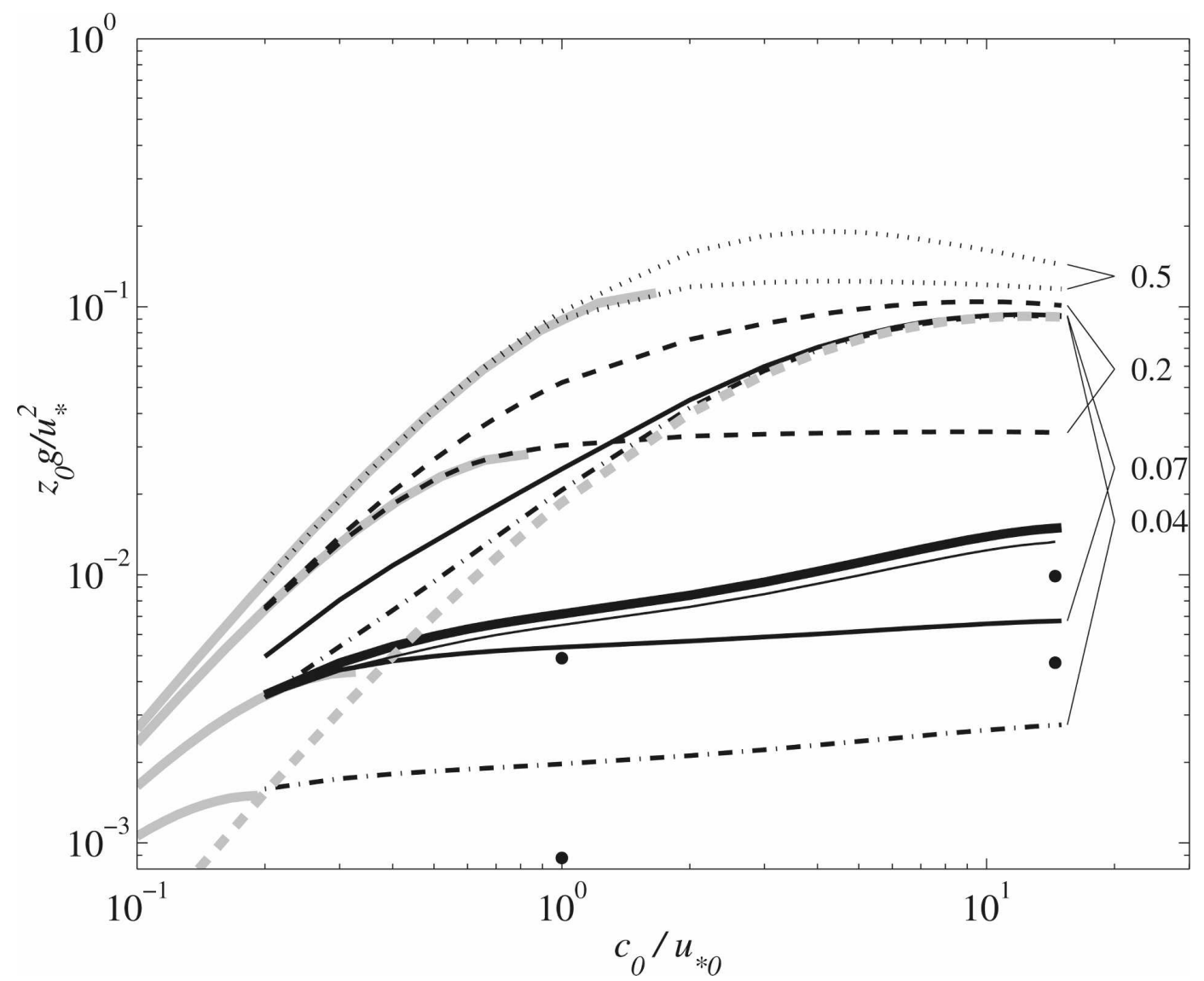

FIG. 6. Modeled Charnock coefficient as function of boundary wave age $c_{0} / u_{* 0}$. Default values of $\mu=0.06$, $B_{\text {sat }}=0.002$, and $\gamma=0.07$ (thick black line), upper and lower limit (medium-width black lines) due to uncertainties in $\mu$ and $B_{\text {sat }}$ for $\gamma=0.04,0.07,0.2,0.5$ (dashed-dotted, solid, dashed, solid, dotted, respectively). Analytic solutions for BO model with same $\gamma$ (solid gray lines: $\gamma$ decreases from top to bottom lines); upper limit for solution without input to breaking waves (dashed gray line), lower limit is $<10^{-4}$ (not shown). With spatial sheltering for $\nu=0.1$ (thin black line) and $\nu=0.4,3.0$ (dots top to bottom at $\left.\mathrm{c}_{0} / u_{* 0}=1,15\right)$ with default parameters $(\mu=0.06$, $\left.B_{\text {sat }}=0.002, \gamma=0.07\right)$.

increases. For the largest $\gamma=0.5$, on the other hand, the input to nonbreaking waves is negligible. In this case, the results converge to the $\mathrm{BO}$ model from KHB at lower wave ages. In all cases, as the wave age decreases, the momentum flux to breaking waves becomes increasingly important. Therefore, the uncertainty of the Charnock coefficient (due to the uncertainty of the spectral level) decreases and the solution converges to the BO model results. Interestingly, our results confirm one of the basic assumptions made by KHB, namely that breaking waves dominate the input to young, strongly forced wave fields.

We will next compare our model results for $\gamma=0.07$ (default value) and previous observations and empirical parameterizations. To do this conveniently, we equate the boundary wave age with the real wave age. Again, we allow the wave height spectrum to vary because we anticipate that $\mu$ and $B_{\text {sat }}$ depend on environmental conditions, such as wind speed and wave age. Although details of the spectral peak have been neglected in our approach, the range of solutions roughly covers the previous observations (Fig. 7). The two empirical formulas fall almost entirely inside the modeled range. For younger wave fields, the model captures well the increase in the Charnock coefficient with wave age. As discussed above, results for very young wave fields are less sensitive to the representation of the wave height spectrum (i.e., the values of $\mu$ and $B_{\text {sat }}$ ), indicating that the drag of very young wave fields is dominated by breaking waves. For very young wave fields the observations are mostly above the upper bound of the NB model results (dashed gray line). This indicates that the input to breaking waves is critical to support the total air-sea momentum flux.

There is some evidence, based on field and laboratory observations (Komen et al. 1996; Donelan et al. 


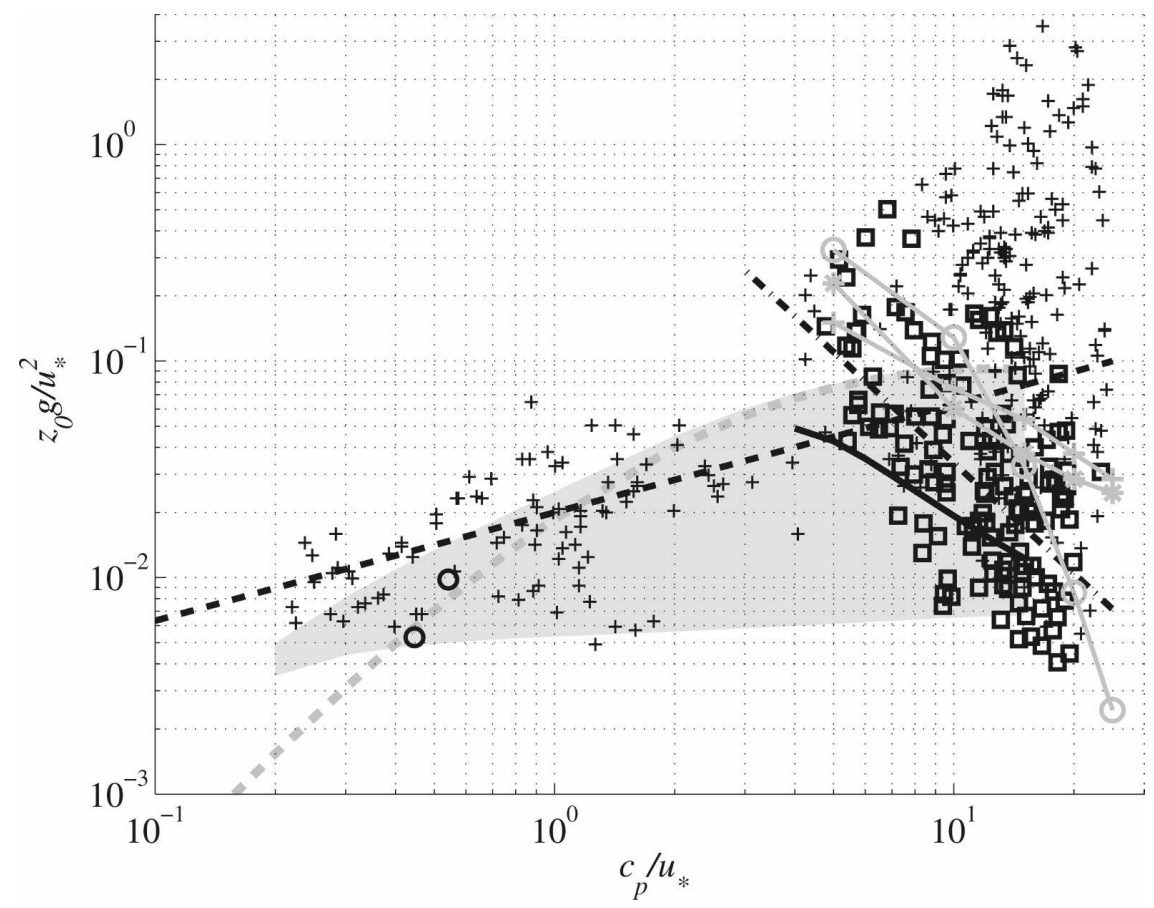

FIG. 7. Observed and modeled Charnock coefficient as function of wave age $c_{p} / u_{*}$ : Range of solutions due to uncertainties in $\mu$ (or $\alpha_{p}$ ) and $B_{\text {sat }}$ for $\gamma=0.07$ (gray area), solution with $B_{\text {sat }}\left(c_{p} / u_{*}\right)$ and $\mu=0.6$ (black thick solid line); upper limit for solutions without input to breaking waves (gray dashed line); previous model results for $u_{*}=0.7 \mathrm{~m} \mathrm{~s}^{-1}$ in solid gray lines from Janssen (1989) (asterisks), Jenkins (1992) (pluses), Jenkins (1993) (circles); data composite for laboratory and field data (Toba and Ebuchi 1991), black pluses; fit to these data (Toba et al. 1990), black dashed line; data based on recent field experiments (squares) and best fit (dashed-dotted line) (Drennan et al., 2003); estimates based on laboratory observations (Donelan et al. 2004) for wind speeds of 30 and $50 \mathrm{~m} \mathrm{~s}^{-1}$ (black circles), where the wave age for the lower wind speed has been estimated using the scaling relation $c_{p} \propto u_{*}^{1 / 3}$ (Jones and Toba 2001).

1985) and numerical modeling results, that $\mu$ and $B_{\text {sat }}$ depend systematically on sea state and wind speed. Within the framework of our model, $\mu$ and $B_{\text {sat }}$ determine the function $\Lambda(B)$. It is physically very sensible that $\Lambda(B)$ depends on sea state and wind speed. For example, $B_{\text {sat }}$ is equivalent to a breaking threshold. Such a threshold is known to depend on environmental conditions because wave breaking is controlled by wave interactions with the turbulent wind, surface currents, and waves of multiple scales (Melville 1996).

Keeping in mind that $\mu$ and $B_{\text {sat }}$ likely depend nontrivially on sea state and wind speed, we will next illustrate how our model can be applied for varying $\mu$ and $B_{\text {sat }}$ to obtain practical wind stress predictions. As an example, we will consider a wave-age-dependent $B_{\text {sat }}$. Based on the analysis of field data, $B_{\text {sat }}$ can be expressed by the empirical formula (Komen et al. 1996)

$$
B_{\text {sat }}=\frac{0.57}{2 \pi}\left(\frac{c_{p}}{u_{*}}\right)^{-3 / 2},
$$

which yields, for fully developed seas with $c_{p} / u_{*}=20$, $B_{\text {sat }}=0.001$, consistent with the lower limit estimated in Part I. These results capture well the enhanced form drag for intermediate wave ages (solid line in Fig. 7). For older to intermediate wave ages, our results are also consistent with previous theoretical estimates, based on the quasi- linear approximation of the hydrodynamic equations neglecting the breaking wave effect (Janssen 1989; Jenkins 1992, 1993). In these previous studies, the one-dimensional wave spectrum was prescribed by the Phillips (1958) spectrum; comparisons in Fig. 7 are made for a spectrum that varies with the same power law in wave age as our wave-age-dependent $B_{\text {sat }}$. The wave-age-dependent $B_{\text {sat }}$ provides an interesting alternative explanation of the enhanced Charnock coefficient at intermediate wave ages to the earlier explanation given by Makin and Kudryavtsev (2002). The model results from Makin and Kudryavtsev indicate that the enhancement at intermediate wave ages is due to the form drag of the dominant breaking wave, which 


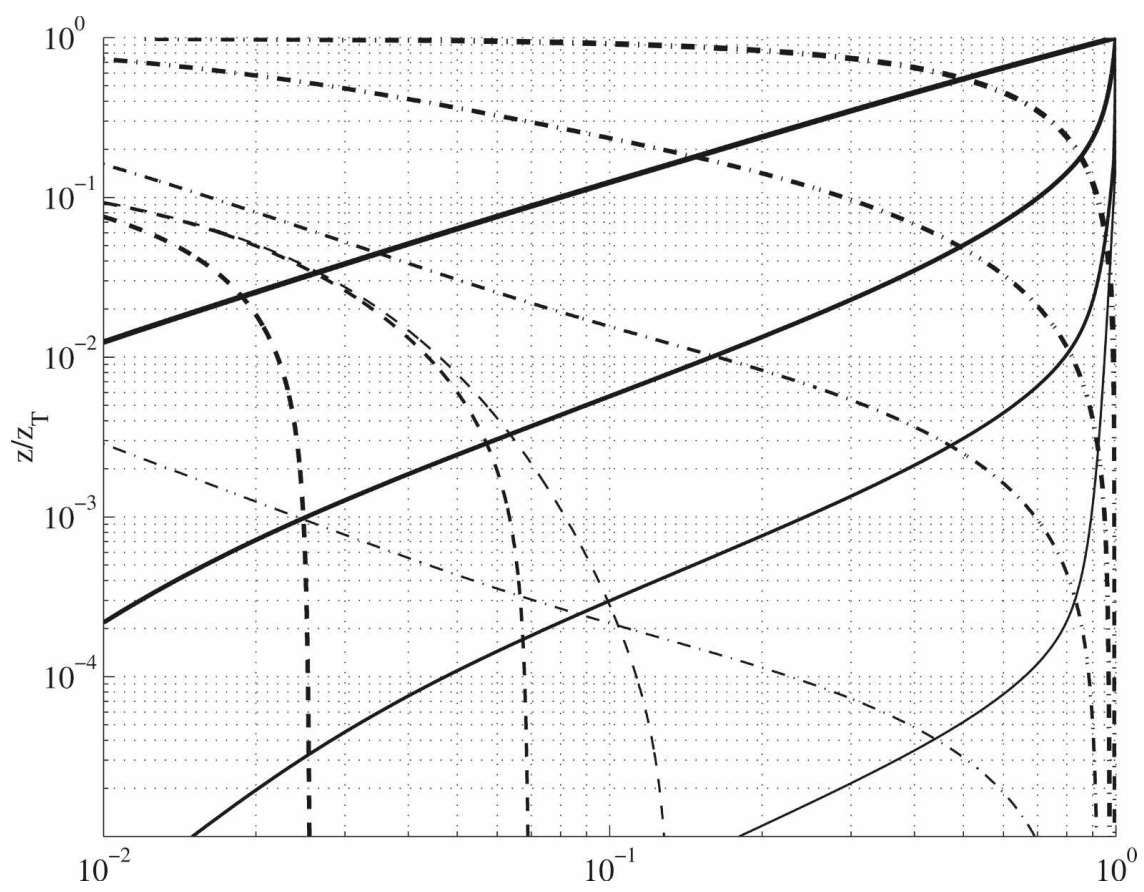

FIG. 8. Stress partitioning as function of height normalized by top of the wave boundary layer, $z_{T}=\epsilon / k_{0}$, for boundary wave ages $0.1,0.5,2,15$ (thick to thin lines): $\tau_{t} / \tau_{0}$ (solid), $\tau_{w} / \tau_{0}$ (dashed), $\tau_{b} / \tau_{0}$ (dashed-dotted); $B_{\text {sat }}=5 \times 10^{-4}$ and default values for other parameters.

was calculated explicitly based on the breaking probability of dominant waves.

Recent observations suggest that the drag coefficient $C_{d}=\left[u_{*} / u(10 \mathrm{~m})\right]^{2}$ decreases for very high wind conditions, for example, under tropical cyclones (Powell et al. 2003; Jarosz et al. 2007). Powell et al. find that $C_{d} \approx$ $2 \times 10^{-3}$ for $u(10 \mathrm{~m})=40 \mathrm{~m} \mathrm{~s}^{-1}$, so $r \approx 0.007$. Within our modeling framework, such a low drag coefficient can be explained by assuming a relatively low high- $k$ spectral level, that is, a low $B_{\text {sat }}$ (see Figs. 6 and 7). Physically, $B_{\text {sat }}$ might be significantly lower for shorter gravity waves at very high winds because the surface wind drift increases with wind speed (see also discussion in Kudryavtsev et al. 1999). The enhanced surface drift, in turn, lowers the slope threshold at which waves break (Banner and Phillips 1974). By the same mechanism, wind gusts could reduce wave heights (Jenkins 2001). In addition, there is some evidence that intense rainfalls, as they occur under hurricanes, dampen the short-wave spectrum (Thorpe 2005). It is interesting to note that our model could explain a reduced drag coefficient under high wind conditions, although the effect of sea spray has been neglected in our approach (for models that include the sea spray effect, see, e.g., Andreas 2004; Makin 2005; Bye and Jenkins 2006; Kudryavtsev 2006).

\section{d. Stress partitioning}

Figures 8 and 9 show how the total stress $\tau_{0}$ partitions into the turbulent stress $\tau_{t}$, the stress due to breaking waves $\tau_{b}$, and nonbreaking waves $\tau_{w}$ at different heights. The results are plotted against the height normalized by the height of the wave boundary layer, $z_{t}=$ $\epsilon / k_{0}$, for different boundary wave ages and two differ-

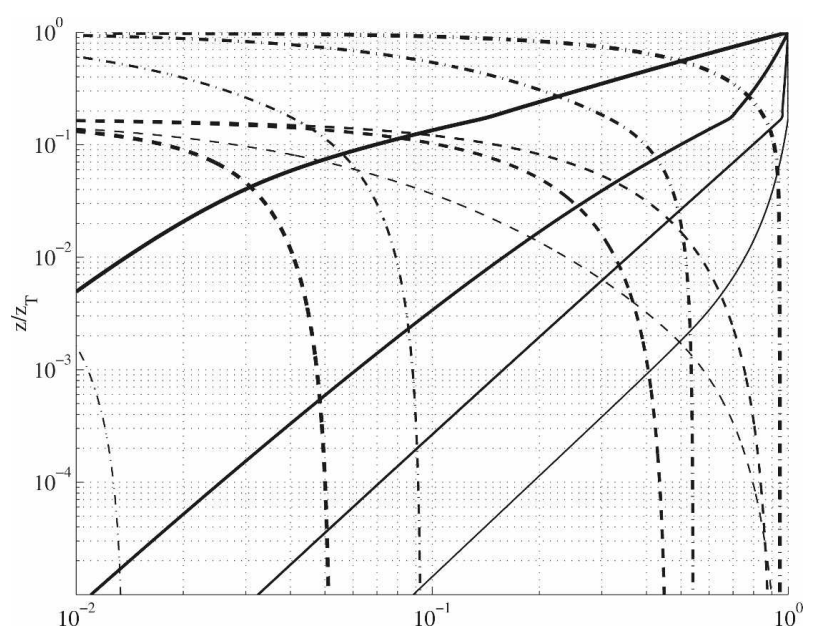

FIG. 9. Stress partitioning for $B_{\text {sat }}=0.01$ and default values for other parameters: line style is identical to Fig. 8. 
ent values (one low and one high) of $B_{\text {sat }}=0.0005,0.01$. Similar to the study by Janssen (1989), the turbulent stress decreases much more rapidly with decreasing height for younger seas close to the top of the wave boundary layer. This is because more energy and momentum is fluxed to waves close to the spectral peak. The result that the contribution of the wave-induced stress increases for younger seas is qualitatively consistent with previous models (Janssen 1989; Jenkins 1992, 1993). Unlike the model by Jenkins (1993), our turbulent stress profiles do not increase linearly with height. Our results suggest that the input to the peak is more important for younger waves and that for older seas the peak is only weakly forced by the wind. Generally, the effect of breaking waves increases for younger seas and lower $B_{\text {sat }}$. For mature seas, the breaking wave stress plays a significant role only if $B_{\text {sat }}$ is low. The lower $B_{\text {sat }}$ is, the smaller the input to nonbreaking waves because of the decreased wave height spectrum. It is interesting to point out that our results are qualitatively consistent with the model from Makin and Kudryavtsev (2002; cf. with their Fig. 2). In particular, both models predict an increase of the breaking stress contribution close to the spectral peak as the wave age decreases.

\section{Spatial sheltering}

We introduce the spatial sheltering effect following KHB so that shorter waves in airflow separation areas of longer breaking waves cannot be forced by the wind. An airflow separation cell extends from the breaking wave crest to a distance, $D=\hat{\epsilon} k^{-1}$, along the wind direction with a height equal to the breaking wave amplitude $a$ (Fig. 10). The constant coefficient $\hat{\epsilon}$ is called the spatial sheltering coefficient. When breaking waves of multiple scales coexist, the fractional horizontal area (area per unit surface area) free of airflow separation, $\alpha(z)$, can be defined: $\alpha \equiv 1$ for heights $z$ outside the wave boundary layer where the airflow is not influenced by breaking waves. In the wave boundary layer, $\alpha(z)$ monotonically decreases with decreasing height if breaking waves are forced by the wind. One may also introduce $\alpha(k)=\alpha(z=\epsilon / k)$, which is the fractional area free of airflow separation due to breaking waves with wavenumbers less than $k$. This function decreases monotonically with increasing $k$. Shorter breaking waves generate new airflow separation regions if they break outside airflow separation regions of longer waves (Fig. 10). Hence, breaking waves at $k$ change $\alpha$ by

$$
\frac{d \alpha}{d k}=-\hat{\epsilon} \alpha(k) \int_{-\pi / 2}^{\pi / 2} \Lambda(k, \theta) \cos \theta d \theta .
$$

\section{a. Governing nondimensional equations}

With spatial sheltering one needs to distinguish between the turbulent Reynolds stress, $\tau_{t}=-\rho_{\alpha}\left\langle u^{\prime} w^{\prime}\right\rangle$ (where $u^{\prime}$ and $w^{\prime}$ are measured outside airflow separation regions, angle brackets denote phase averaging), and the "effective" turbulent stress, $\alpha \tau_{t}$, which is the average downward transport of horizontal momentum due to turbulent eddies. The wave stress is $\tau_{w}=-\rho_{\alpha}\langle\tilde{u} \tilde{w}\rangle$, where $\tilde{u}$ and $\tilde{w}$ are measured outside airflow separation regions so that the effective wave stress is $\alpha \tau_{w}$. Air-side momentum conservation now dictates

$$
\tau_{0}=\alpha(z) \tau_{t}(z)+\alpha(z) \tau_{w}(z)+\tau_{b}(z)=\text { const. }
$$

The wave-induced momentum flux at height $z$ into nonbreaking waves is expressed as

$$
\alpha(z) \tau_{w}(z)=\int_{0}^{\infty} \int_{-\pi / 2}^{\pi / 2} \alpha(z=\delta / k) \rho_{w} \omega \beta_{g}(k, \theta) \Psi(k, \theta) F(z, k) \cos \theta k d \theta d k,
$$

where $\omega$ is the wave frequency and $F(z, k)$ denotes a decay function to account for the decrease in waveinduced stress with height, which is approximated by a step function. The factor $\alpha \leq 1$ assures that wind forcing of waves is reduced due to the sheltering of airflow separation regions. The growth rate of wind-driven gravity waves, $\beta_{g}$, is given by

$$
\beta_{g}(k, \theta)=s_{\beta} c_{\beta} \omega \frac{\tau_{t}(z=\delta / k)}{\rho_{\omega} c^{2}} h_{\beta}(\theta)
$$

With spatial sheltering the form drag of breaking waves changes to (cf. Part I)

$$
\begin{aligned}
\tau_{b}(z)= & \int_{0}^{\epsilon / z} \int_{-\pi / 2}^{\pi / 2} \rho_{a} s_{p} \beta_{\Lambda} \Lambda(k, \theta) \alpha(k) \\
& \times(u(k) \cos \theta-c)^{2} \cos \theta d \theta d k .
\end{aligned}
$$

Similarly, the two-dimensional spectral energy flux to breaking waves at $k$ (corresponding to height $\epsilon k^{-1}$ ) is

$$
I_{b}(k, \theta)=\rho_{a} s_{p} \beta_{\Lambda} \alpha(k) \Lambda(k, \theta) k^{-1} c[u(k) \cos \theta-c]^{2}
$$

and to nonbreaking waves (corresponding to the inner layer height $\delta k^{-1}$ ) it is

$$
I_{w}(k, \theta)=c_{\beta} s_{\beta} h_{\beta}(\theta) k^{-2} c \alpha(z=\delta / k) \tau_{t}(z=\delta / k) B(k, \theta) .
$$



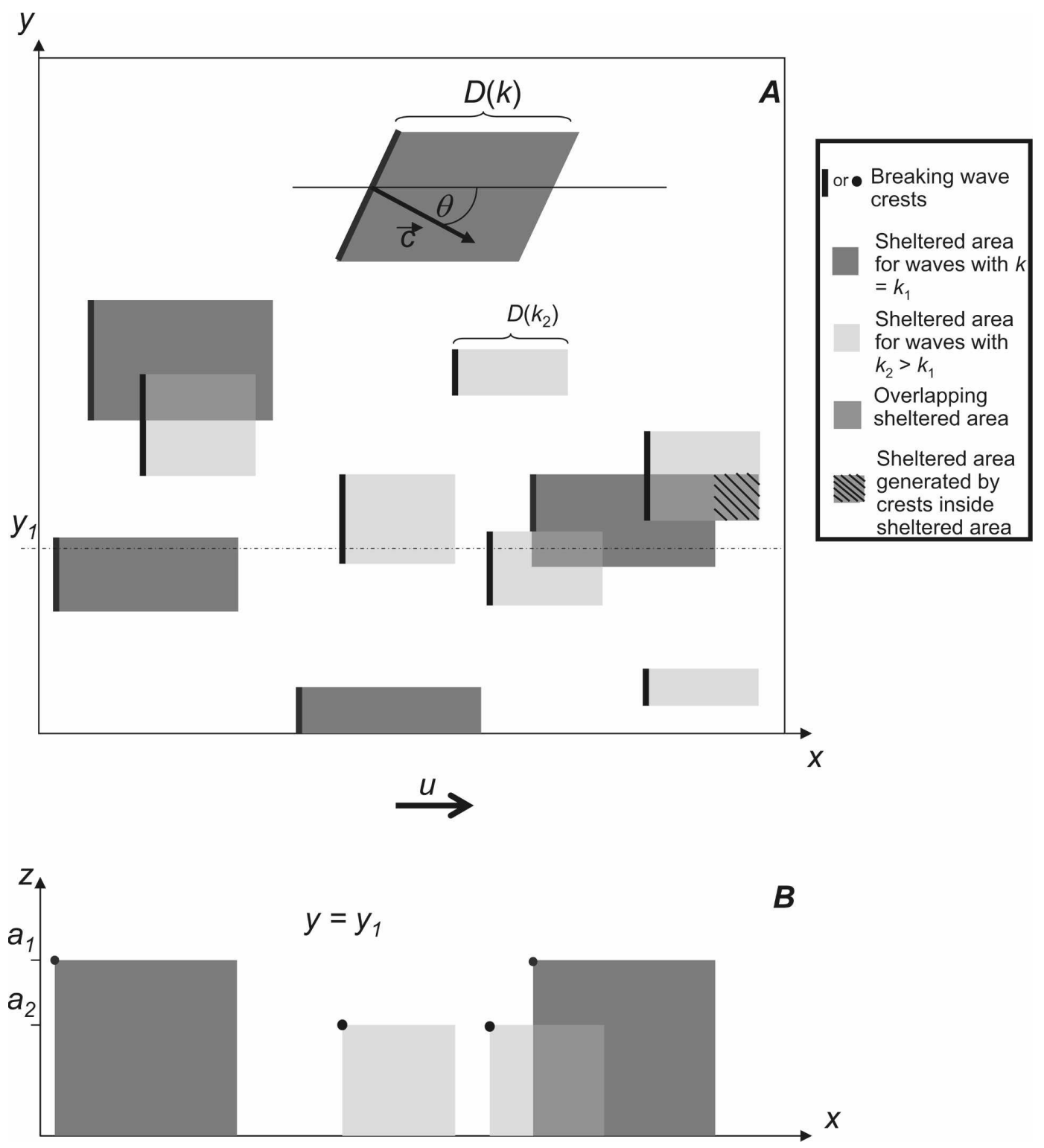

FIG. 10. Illustration of airflow separation regions (sheltered area) for waves at two different wavenumbers: (a) bird's-eye view and (b) cross section at $y=y_{1}$.

Since spatial sheltering causes a reduction of the effective air space, the flux terms as well as loss terms must also be reduced by a factor $\alpha$ in the air-side energy balance,

$$
\frac{d\left[\alpha u\left(\tau_{t}+\tau_{w}\right)\right]}{d_{z}}-\alpha \varepsilon-\frac{d\left[\alpha \Pi_{w}\right]}{d_{z}}-e_{b}=0,
$$

where the energy transfer terms are

$$
\alpha(z) \Pi_{w}(z)=-\int_{0}^{\delta / z} \int_{-\pi / 2}^{\pi / 2} I_{w}(k, \theta) k d \theta d k
$$

and

$$
e_{b}(z) d z=-\int_{-\pi / 2}^{\pi / 2} I_{b}(k, \theta) k d \theta d k
$$

and the loss term $\varepsilon$ due to dissipation of turbulent kinetic energy is

$$
\varepsilon=\frac{\tau_{t}^{3 / 2}}{\rho_{a}^{1 / 2} \kappa z} .
$$

It is convenient to combine the governing air-side equations and to introduce the nondimensional variables and model coefficients from Part I. The normal- 
ized turbulent and nonbreaking wave stress is now, respectively, defined by

$$
S=\frac{\alpha \tau_{t}}{\rho_{a} c^{2}}
$$

and

$$
S_{w}=\frac{\alpha \tau_{w}}{\rho_{a} c^{2}}
$$

Furthermore, we introduce the model spatial sheltering coefficient (same as the $\delta$ coefficient in KHB) as

$$
\nu=\frac{\hat{\epsilon}}{b^{\prime}}=\frac{\hat{\epsilon} \rho_{a}}{b \rho_{w}} .
$$

The resulting system of nondimensional equations closely resembles the one from Part I:

$$
\begin{aligned}
& \frac{d S}{d K}=S-M_{b}-M_{w}, \\
& \frac{d U}{d K}=\frac{1}{2} U-\left(S+S_{w}\right)^{-1}\left(E_{b}+\delta_{\epsilon}^{-1 / 2} E_{w}-U M_{b}+\kappa^{-1} \alpha^{-1 / 2} S^{3 / 2}\right), \\
& \frac{d \alpha}{d K}=-\nu \gamma^{-2} \alpha \int_{-\pi / 2}^{\pi / 2}\left[k \beta_{\Lambda} \Lambda(K, \theta)\right] \cos \theta d \theta, \\
& \frac{d S_{w}}{d K}=S_{w}+M_{w} .
\end{aligned}
$$

The normalized flux terms in the governing nondimensional equations are

$$
M_{b}=\int_{-\pi / 2}^{\pi / 2} \alpha s_{p}\left[k \beta_{\Lambda} \Lambda(k, \theta)\right][U \cos \theta-1]^{2} \cos \theta d \theta
$$

$$
\begin{aligned}
M_{w} & =S \int_{-\pi / 2}^{\pi / 2} s_{\beta} c_{\beta} B(K-\Delta, \theta) h_{\beta}(\theta) \cos \theta d \theta, \\
E_{b} & =\int_{-\pi / 2}^{\pi / 2} \alpha s_{p}\left[k \beta_{\Lambda} \Lambda(K, \theta)\right][U \cos \theta-1]^{2} d \theta,
\end{aligned}
$$

$$
E_{w}=S \int_{-\pi / 2}^{\pi / 2} s_{\beta} c_{\beta} B(k-\Delta, \theta) h_{\beta}(\theta) d \theta .
$$

From the wave energy equation

$$
I_{w}(k, \theta)+I_{b}(k, \theta)+N L(k, \theta)-D(k, \theta)=0,
$$

and the relation $\Lambda(B)$,

$$
\Lambda(k, \theta)=\gamma_{\Lambda} b^{-1} k^{-1}[B(k, \theta)]^{3}\left[1+\left(\frac{B(k, \theta)}{B_{\text {sat }}}\right)^{n}\right],
$$

with $n \gg 1$ and $\gamma_{\Lambda}=\mu_{\Lambda}^{-2}\left(\rho_{a} / \rho_{w}\right) c_{\beta}^{3}$ (cf. Part I), one further obtains expressions for $B$ and $\Lambda$ in nondimensional variables.

For $B(K, \theta)<B_{\text {sat }}$ and $s_{\beta}=1$,

$$
\begin{aligned}
c_{\beta} B(K, \theta) & =\left[\delta_{\epsilon} S(K+\Delta) \frac{h_{\beta}(\theta)}{\mu^{-2}-\gamma^{2} \mu_{\Lambda}^{-2} \alpha s_{p}(U \cos \theta-1)^{2}}\right]^{1 / 2}, \\
k \beta_{\Lambda} \Lambda(k, \theta) & =\gamma^{2} \mu_{\Lambda}^{-2}\left[c_{\beta} B(K, \theta)\right]^{3} .
\end{aligned}
$$

For $B(K, \theta) \rightarrow B_{\text {sat }}$,

$$
\begin{aligned}
c_{\beta} B(K, \theta) & =c_{\beta} B_{\text {sat }}, \\
k \beta_{\Lambda} \Lambda(k, \theta) & =\frac{\gamma^{2} \delta_{\epsilon} h_{\beta}(\theta) s_{\beta} S(K+\Delta)+\gamma^{2}\left(\mu_{\Lambda}^{-2}-\mu^{-2}\right)\left(c_{\beta} B_{\text {sat }}\right)^{2}}{1-\gamma^{2} \alpha s_{p}[U \cos \theta-1]^{2}} c_{\beta} B_{\text {sat }} .
\end{aligned}
$$

As in the case without spatial sheltering, solving the system of equations from $k_{0}$ to $k_{1}$ (or $K_{0}$ to $K_{1}$ ) requires specification of the model coefficients as well as boundary conditions (including the delay and advance terms outside the domain of integration). We assume that the spatial sheltering effect closer to the peak is weak so that $\alpha=1$ at height $\epsilon / k_{0}$ and $\alpha=1$ for all $k<k_{0}$. In principle, $\hat{\epsilon}$ can assume values from zero (no spatial 

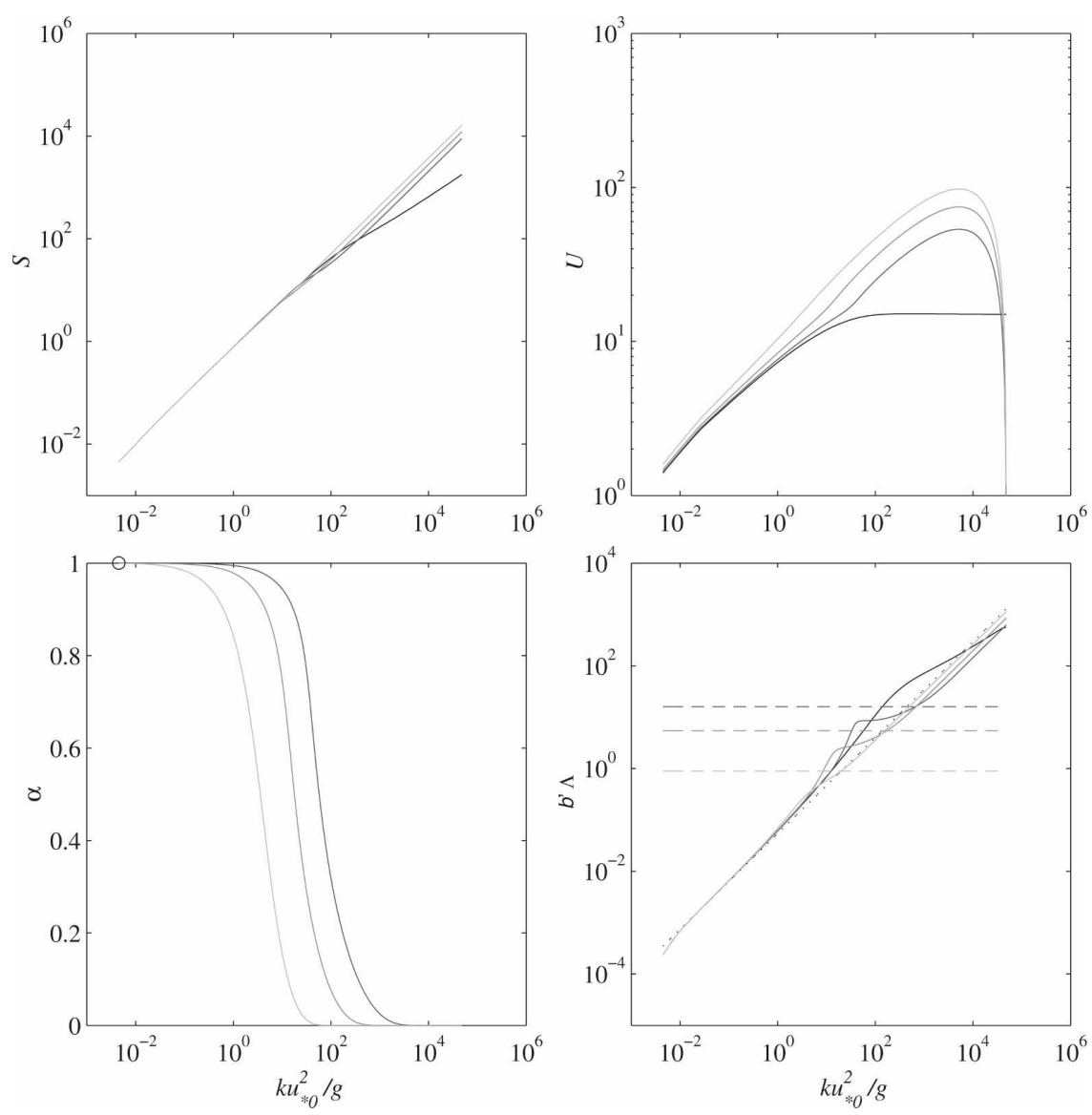

FIG. 11. Spatial sheltering solutions for $c_{0} / u_{* 0}=15$ with $\nu=0.0$ (black), $\nu=0.1$ (dark gray), $\nu=0.4$ (gray), $\nu=3$ (light gray), and $B_{\text {sat }}=0.002$ : Numeric solutions (solid lines) for $S, U$, $\alpha$, and $b^{\prime} \Lambda$; asymptotic solution for nonbreaking waves (A11) with same $c_{0} / u_{* 0}$ (dotted line), asymptotic high- $k$ limit with spatial sheltering effect from KHB (dashed lines).

sheltering) to $2 \pi$ (airflow separation area extends from wave crest to wave crest). Based on laboratory observations by Reul et al. (1999), we assume here by default that the airflow separation cell extends from the wave crest to trough so that $\hat{\epsilon}=\pi$. With $b^{\prime}=8$ (corresponding to our default $b=b^{\prime} \rho_{a} / \rho_{w}=0.01$; see Part I) this results in $\nu=\hat{\epsilon} / b^{\prime} \approx 0.4$. For the upper bound of $b^{\prime}$ and the default $\hat{\epsilon}$, one obtains $\nu \approx 0.1$. Because these parameters are not well constrained, however, and to investigate strong spatial sheltering, we also consider the greatest possible value of $\nu \approx 3(\hat{\epsilon}=2 \pi$ and smallest $b^{\prime}=2$, see KHB).

\section{b. Results}

We will first illustrate that for the default value of $B_{\text {sat }}=0.002$, spatial sheltering can significantly alter the wind and wave dynamics. Figures 11 and 12 show results for boundary wave ages of 15 and 1 , respectively, and $\nu=0,0.1,0.4,3.0$, as discussed above. The satura- tion spectrum $B$ is not shown as it is hardly influenced by spatial sheltering and because $B$ converges quickly to its limiting-value $B_{\text {sat }}$ and remains constant. Note that for high wind speeds and short surface waves, Kudryavtsev and Makin (2007) attribute a decrease in microwave backscatter response, which has been observed by Donelan et al. (2004), to the spatial sheltering effect. In section $3 c$ we discuss different mechanisms that could be responsible for such a decrease in backscatter response due to a reduced $B$.

The normalized turbulent stress $S$ (top left) is higher for greater $\nu$, indicating that the momentum input to the wave field is reduced. The normalized wind speed profile is notably altered (top right) once a significant air fraction is covered by airflow separation regions (bottom left). For mature seas, the spatial sheltering effect is only important for shorter waves where it influences the number of breaking waves (bottom right, Fig. 11). For younger seas, spatial sheltering also affects waves closer to the spectral peak (Fig. 12). The break- 

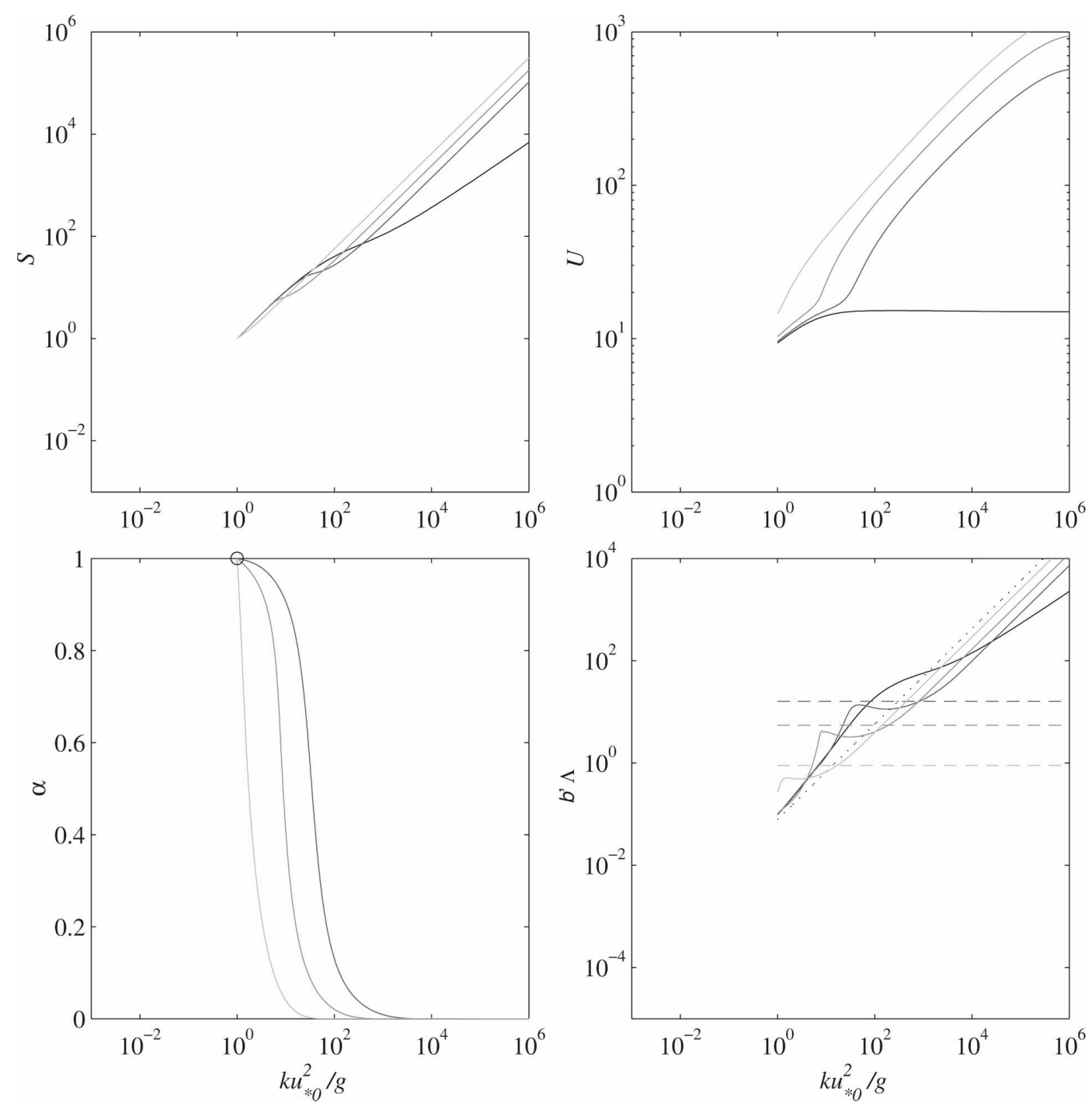

FIG. 12. As in Fig. 11, but for $c_{0} / u_{* 0}=1$.

ing wave distribution first approaches the asymptotic high- $k$ limit for the BO model of KHB (dashed lines in bottom right panel). For higher $k$, however, spatial sheltering changes the asymptotic high- $k$ limit from the BO solution to a nonbreaking solution (shown as dotted black line).

Figure 6 shows the effect of spatial sheltering on the Charnock coefficient. With a weak sheltering effect $(\nu=0.1)$, the Charnock coefficient is only slightly reduced. The difference disappears for very young seas, which is consistent with the BO model (KHB) that predicts no spatial sheltering effect on the Charnock coefficient. This is because most of the momentum and energy is transferred to waves closer to the spectral peak for very young seas.

With a larger $\nu$ numerical solutions cannot be ob- tained for very young seas. Note that KHB also found a theoretical lower limit for the boundary wave age, such that $c_{0} / u_{* 0}>\sqrt{\nu / 2}$ when the spatial sheltering effect was included. Therefore, it might not be trivial to investigate the sheltering effect on very young wave fields with boundary wave ages much lower than this threshold. For wave ages above one, the larger the spatial sheltering effect is, the smaller the normalized roughness length. However, only with the greatest possible spatial sheltering coefficient of $\nu=3.0$ does the Charnock coefficient decrease significantly (up to a factor of $\sim 10$; see Fig. 6 ).

Figures 13 and 14 show how the stress partitioning is affected by spatial sheltering for boundary wave ages of 15 and 1, respectively. Spatial sheltering modifies the stress partitioning close to the surface; it reduces the 


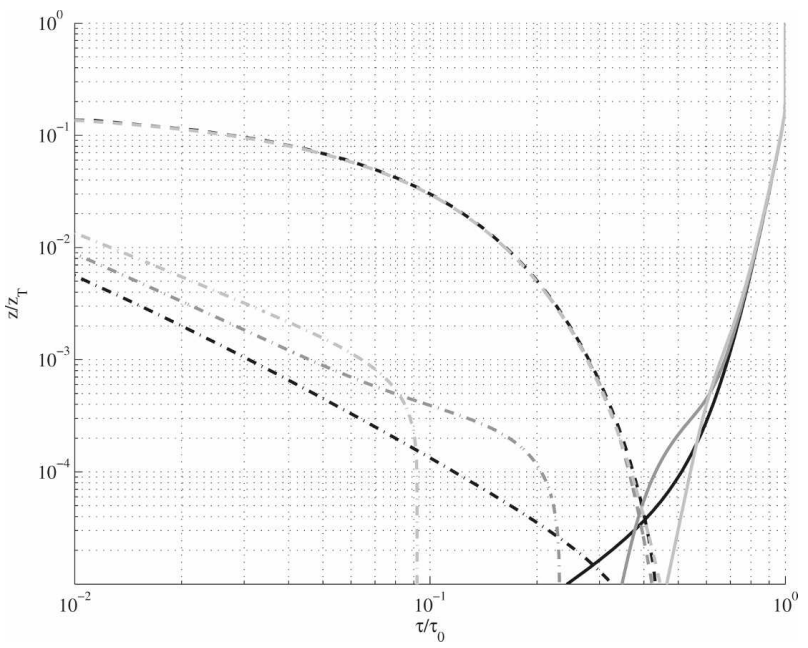

FIG. 13. Stress partitioning as function of height normalized by top of the wave boundary layer for $\nu=0.0$ (black), $\nu=0.4$ (dark gray), $\nu=3$ (light gray), $c_{0} / u_{* 0}=15$, and $B_{\text {sat }}=0.002: \tau_{t} / \tau_{0}$ (solid), $\tau_{w} / \tau_{0}$ (dashed), $\tau_{b} / \tau_{0}$ (dashed-dotted).

contribution of breaking waves and increases the turbulent stress contribution. Notice also that the spatial sheltering effect is much stronger for younger waves.

We have also investigated the sheltering effect for different values of $B_{\text {sat. }}$. If $B_{\text {sat }}$ is relatively large ( $B_{\text {sat }}>$ 0.01 ), spatial sheltering affects little the overall wind and wave dynamics and results are similar to the nospatial-sheltering results. Potentially, spatial sheltering might still influence other air-side processes, such as scalar fluxes, since the air space available for scalar transport might still be significantly reduced.

\section{Conclusions}

We have developed a coupled wind and wave model. The conservation of air-side momentum and energy, as

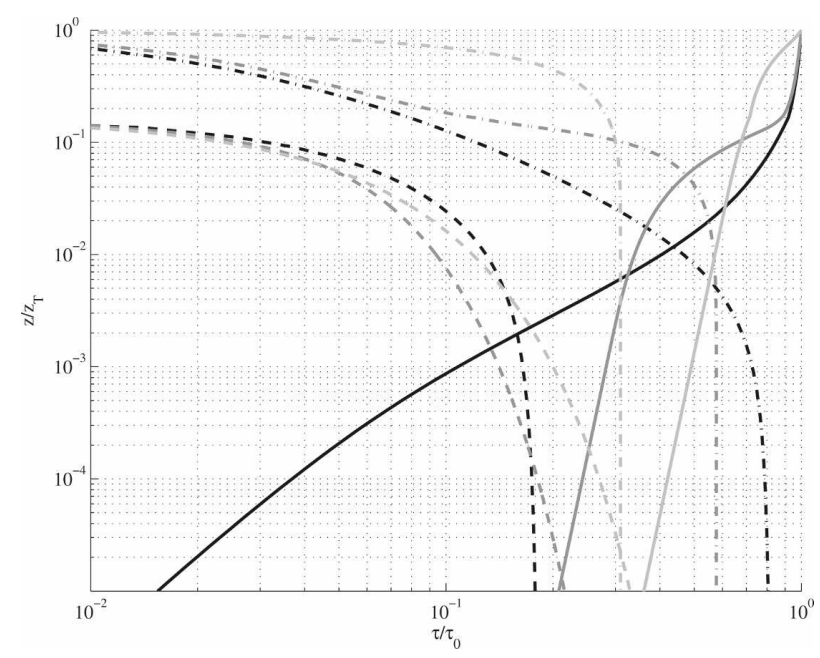

FIG. 14. As in Fig. 13, but for $c_{0} / u_{* 0}=1$. well as wave energy, leads to a coupled system of nonlinear advance-delay differential equations for the wave height spectrum, the breaking wave distribution, and the two air-side profiles of wind speed and turbulent stress. The system of equations was closed by introducing a relation between the breaking wave distribution $\Lambda$ and the wave height curvature spectrum $B$ such that for a threshold of $B$, called the "threshold saturation-level" $B_{\text {sat }}$ (Alves and Banner 2003), the number of breaking waves strongly increases.

In the companion paper (Part I), the model was applied for mature seas. In this study, we have applied the model to the equilibrium range under a variety of conditions, including very young, strongly forced laboratory waves to mature ocean wave fields. In particular, we have introduced an idealized growing-sea model by extending our model to the spectral peak. Consistent with model results by Makin and Kudryavtsev (2002), the input to breaking waves continuously increases with inverse wave age until the input to the spectral peak is dominated by breaking waves. For low $B_{\text {sat }}$ the major wind input to shorter waves away from the spectral peak spectrum is determined by breaking waves. If $B_{\text {sat }}$ increases, however, the wind input is dominated by nonbreaking waves. Therefore, model results depend sensitively on the value of $B_{\text {sat }}$.

In general, if breaking waves dominate the wind input, the solution converges to the breaking-only or breaking-dominated model result. The one-dimensional breaking wave distribution, $\Lambda(k)$, as a function of wavenumber $k$ converges to a constant in the BO model and approaches asymptotically $k^{2 / 3}$ in the BD model. If, on the other hand, nonbreaking waves dominate the wind input, the solution converges to the nonbreaking model result and $\Lambda(k)$ varies as power law in $k$ with an exponent that depends on the threshold saturation level.

Our model results at intermediate to large wave ages are generally consistent with previous field observations. In particular, the model suggests that the observed decrease in the Charnock coefficient (normalized roughness length) with wave age for moderate wind conditions may be due to a decrease of the threshold saturation level with wave age. Our model results are also consistent with laboratory observations at lower wave ages, which indicate an increase in normalized roughness length with wave age. The low drag coefficient observed for high wind hurricane conditions could be explained by assuming a relatively low threshold saturation level.

Finally, we have introduced the spatial sheltering effect so that shorter waves in airflow separation regions of breaking longer waves cannot be forced by the wind. 
Spatial sheltering always causes a significant reduction in air space available for transport processes through the air-sea interface. For lower values of $B_{\text {sat }}$ spatial sheltering also strongly modifies the coupled wind and wave dynamics. However, the sheltering effect on the Charnock coefficient is generally small; it becomes significant only if the largest possible sheltering effect is imposed.

In summary, the effect of breaking waves plays a significant role and needs to be incorporated so as to model coupled wind and wave dynamics more realistically. Clearly, more observations of the breaking wave distribution, energy dissipation of breaking waves, and form drag of breaking waves are needed to better constrain our model parameters. Furthermore, for practical air-sea momentum flux predictions, it is critical to understand the dependence of the short-wave spectrum on environmental conditions, such as sea state and wind speed.

Acknowledgments. We thank two anonymous reviewers for their helpful comments and suggestions, which improved the manuscript. This work was supported by the U.S. National Science Foundation (Grant OCE- 0526177) and the U.S. Office of Naval Research (Grant N00014-06-10729).

\section{APPENDIX}

\section{Solutions with Wind Input to Either Breaking or Nonbreaking Waves}

As discussed in Part I, if the input to breaking waves is weak (strong), the full model converges to the simplified models without any input to breaking (nonbreaking) waves. To understand better the full model, we will therefore briefly discuss these simplified cases. Throughout this appendix the spatial sheltering effect is not included for simplicity.

\section{a. Nonbreaking solutions}

The model without wind input to breaking waves was previously derived by Hara and Belcher $(2002,2004)$.
These models, however, did not introduce the saturation limit $B_{\text {sat. }}$. Here, we will focus on how introducing the limiting $B_{\text {sat }}$ will change the model results by Hara and Belcher $(2002,2004)$.

Let us first introduce variables evaluated at the inner layer height $z=\delta / k$ instead of the breaking wave height $z=\epsilon / k$ so that $K \rightarrow K+\Delta, S \rightarrow \delta_{\epsilon} S$, and $U \rightarrow \delta_{\epsilon}^{1 / 2} U$. Then, the equations for the normalized stress and normalized wind speed become

$$
\begin{aligned}
& \frac{d S}{d K}=S-M_{w}, \\
& \frac{d U}{d K}=\frac{1}{2} U-S_{0}^{-1} \exp (-K)\left(E_{w}+\kappa^{-1} S^{3 / 2}\right) .
\end{aligned}
$$

The normalized flux terms in the governing nondimensional equations are

$$
\begin{aligned}
M_{w} & =S \int_{-\pi / 2}^{\pi / 2} s_{\beta} c_{\beta} B(k, \theta) h_{\beta}(\theta) \cos \theta d \theta, \\
E_{w} & =S \int_{-\pi / 2}^{\pi / 2} s_{\beta} c_{\beta} B(k, \theta) h_{\beta}(\theta) d \theta .
\end{aligned}
$$

Furthermore, for $B(K, \theta)<B_{\text {sat }}$ and $s_{\beta}=1$

$$
c_{\beta} B(K, \theta)=\mu\left[S(K) h_{\beta}(\theta)\right]^{1 / 2},
$$

and for $B(K, \theta) \rightarrow B_{\text {sat }}$

$$
c_{\beta} B(K, \theta)=c_{\beta} B_{\text {sat }} .
$$

The breaking distribution is given by

$$
k b^{\prime} \Lambda(k, \theta)=s_{\beta} S(K)\left[c_{\beta} B(K, \theta)\right] h_{\beta}(\theta) .
$$

Without $B_{\text {sat }}, c_{\beta} B(k, \theta)$ converges to $8 /(3 \pi)$ for $k \rightarrow \infty$ due to spectral sheltering. Therefore, for any $c_{\beta} B_{\text {sat }}>$ $8 /(3 \pi), B_{\text {sat }}$ does not affect the solutions at high $k$. For $B_{\text {sat }}>B(k, \theta)$, the saturation spectrum and distribution of breaking waves are, respectively, given by

$$
B(K, \theta)=\frac{8}{3 \pi} \frac{1}{c_{\beta}}\left[1+\frac{8 S_{0}^{-1 / 2}-3 \pi \mu}{3 \pi \mu}\left(\frac{k_{0}}{k}\right)^{1 / 2}\right]^{-1} \cos \theta
$$

and

$$
k b^{\prime} \Lambda(k, \theta)=\mu_{\Lambda}^{-2}\left(\frac{8}{3 \pi}\right)^{3}\left[1+\frac{8 S_{0}^{-1 / 2}-3 \pi \mu}{3 \pi \mu}\left(\frac{k_{0}}{k}\right)^{1 / 2}\right]^{-3} \cos ^{3} \theta
$$

If, on the other hand, $c_{\beta} B_{\text {sat }}<8 /(3 \pi), B(k, \theta)$ converges to $B_{\text {sat }}$ at high $k$. At lower wavenumbers where $B<B_{\text {sat }}$ the above solutions are still valid. Asymptotic solutions for $B \rightarrow B_{\text {sat }}$ are found by solving (A1) with (A3) and (A6). Then, the turbulent stress is given by

$$
\tau_{t}=\tau_{0}\left(\frac{k}{k_{0}}\right)^{-(4 / 3) c_{\beta} B_{\mathrm{sat}}}
$$

From the wave energy balance (A7), $\Lambda$ is determined to be 


$$
k b^{\prime} \Lambda(k, \theta)=S_{0}\left[c_{\beta} B_{\text {sat }}\right] h_{\beta}(\theta)\left(\frac{k}{k_{0}}\right)^{1-(4 / 3) c_{\beta} B_{\text {sat }}} .
$$

Interestingly, the last result suggests that the power law behavior of the breaking distribution is not universal but depends on the spectral level.

\section{b. Breaking-only and breaking-dominated solutions}

KHB obtained the solution with wind input to breaking waves only (BO solution). The full model solution indeed converges to this limit if the wind input to nonbreaking waves is small over the entire wavenumber range. If, on the other hand, the input to nonbreaking waves is small at higher wavenumbers but is significant at low wavenumbers, the full model solution at higher wavenumbers converges to a different asymptotic solution (breaking-dominated solution) as described below.

We assume that breaking waves dominate the input to waves for all $k>k_{a}$ and $S_{a}=S\left(k_{a}\right)$. It is further assumed that the nonbreaking wave stress remains constant at $\tau_{w}\left(k_{a}\right)$ for all $k>k_{a}$. If breaking waves are dominant, $\Lambda$ is approximately unidirectional (see Part I), so the wave energy balance becomes

$$
M_{b}=b^{\prime} \Lambda(k)
$$

and $M_{b}=E_{b}$. The governing normalized equations are given by

$$
\begin{aligned}
\frac{d S}{d K} & =S-M_{b}, \\
0 & =\frac{1}{2} U\left(S+S_{w}\right)+M_{b}(U-1)-\kappa^{-1} S^{3 / 2}, \\
U & =1+\gamma^{-1}, \\
\frac{d S_{w}}{d K} & =S_{w} .
\end{aligned}
$$

Note that, although flux terms to nonbreaking waves are negligible, the normalized wave-induced stress $S_{w}$ may not be, as longer nonbreaking waves could have induced a significant stress, which remains constant at high $k$ and influences the energy flux term $u\left(\tau_{t}+\tau_{w}\right)$. A combination of the last equations yields

$$
\frac{d S}{d K}=\frac{3+\gamma}{2} S-\frac{\gamma}{\kappa} S^{3 / 2}+\frac{1+\gamma}{2} S_{w} .
$$

We may distinguish two cases:

1) $S \gg S_{w}$ or $\tau_{t} \gg \tau_{w}$ (BO limit)

The nonbreaking-wave stress is small compared to the turbulent stress. We recover the $\mathrm{BO}$ model by $\mathrm{KHB}$, for which

$$
k b^{\prime} \Lambda(k, \theta)=\left(\kappa \frac{3+\gamma}{2 \gamma}\right)^{2 / 3} \delta(\theta) .
$$

2) $S \ll S_{w}$ or $\tau_{t} \ll \tau_{w}$ (BD limit)

This case will always occur in the asymptotic limit of large $k$, if there is a finite flux to nonbreaking waves. This is because the breaking wave stress increases and $\tau_{t}$ goes to zero at large wavenumbers, but $\tau_{w}$ remains finite. The governing equation simplifies to

$$
\frac{d S}{d K}=-\frac{\gamma}{\kappa} S^{3 / 2}+\frac{1+\gamma}{2} S_{w}
$$

For $\tau_{w}=$ const

$$
S_{w}=\frac{\tau_{w}}{\rho_{a} c^{2}}=\frac{\tau_{w}}{\tau_{0}} \frac{u_{*}^{2}}{c_{0}^{2}} \frac{k}{k_{0}}=\Gamma \frac{k}{k_{0}},
$$

where $\Gamma$ is a constant coefficient. Substitution results in

$$
\frac{d S}{d k}=-\frac{\gamma}{\kappa} k^{-1} S^{3 / 2}+\frac{1+\gamma}{2} \Gamma,
$$

where $k$ now is the wavenumber normalized by $k_{0}$. We define

$$
S \equiv k^{2 / 3} q
$$

so that

$$
k^{2 / 3} \frac{d q}{d k}=-\frac{\gamma}{\kappa} q^{3 / 2}+\frac{1+\gamma}{2} \Gamma-\frac{2}{3} k^{-1 / 3} q .
$$

Because

$$
k^{-1 / 3} q=\frac{S}{k} \propto \frac{S}{S_{w}} \ll 1,
$$

we may neglect the last term, and the governing equation becomes

$$
\frac{d q}{d\left(3 k^{1 / 3}\right)}=-\frac{\gamma}{\kappa} q^{3 / 2}+\frac{1+\gamma}{2} \Gamma .
$$

Critical-point analysis shows that there is a stable node at

$$
q_{c}=\left(\frac{1+\gamma}{2 \gamma} \kappa \Gamma\right)^{2 / 3} .
$$

Therefore, the asymptotic solutions $S$ and $\Lambda$ for $k \rightarrow$ $\infty$ are

$$
\begin{aligned}
S & =\left(\frac{1+\gamma}{2 \gamma} \kappa \Gamma\right)^{2 / 3} k^{2 / 3}, \\
b^{\prime} \Lambda(k) & =\frac{1}{3}\left(\frac{1+\gamma}{2 \gamma} \kappa \Gamma\right)^{2 / 3} k^{2 / 3} .
\end{aligned}
$$

The breaking-wave distribution is influenced by the presence of a nonbreaking wave stress and is proportional to $k^{2 / 3}$ at large wavenumbers. 
These simple asymptotic solutions are helpful in interpreting the results of the more complex full model with input to both breaking and nonbreaking waves.

\section{REFERENCES}

Alves, J. H. G. M., and M. L. Banner, 2003: Performance of a saturation-based dissipation-rate source term in modeling the fetch-limited evolution of wind waves. J. Phys. Oceanogr., 33, 1274-1298.

Andreas, E. L., 2004: Spray stress revisited. J. Phys. Oceanogr., 34, $1429-1440$.

Banner, M. L., 1990: The influence of wave breaking on the surface pressure distribution in wind-wave interactions. J. Fluid Mech., 211, 463-495.

_ , and O. M. Phillips, 1974: On the incipient breaking of small scale waves. J. Fluid Mech., 65, 647-656.

_ I. S. F. Jones, and J. C. Trinder, 1989: Wavenumber spectra of short gravity waves. J. Fluid Mech., 198, 321-344.

Belcher, S. E., and J. C. R. Hunt, 1998: Turbulent flow over hills and waves. Annu. Rev. Fluid Mech., 30, 507-538.

Bye, J. A. T., and A. D. Jenkins, 2006: Drag coefficient reduction at very high wind speeds. J. Geophys. Res., 111, C03024, doi:10.1029/2005JC003114.

Cavaleri, L., 2006: Wave modeling: Where to go in the future. Bull. Amer. Meteor. Soc., 87, 207-214.

Donelan, M. A., J. Hamilton, and W. H. Hui, 1985: Directional spectra of wind-generated waves. Philos. Trans. Roy. Soc., London, A315, 509-562.

—, B. K. Haus, N. Reul, W. J. Plant, M. Stiassnie, H. C. Graber, O. B. Brown, and E. S. Saltzman, 2004: On the limiting aerodynamic roughness of the ocean in very strong winds. Geophys. Res. Lett., 31, L18306, doi:10.1029/2004GL019460.

Drennan, W. M., H. C. Graber, D. Hauser, and C. Quentin, 2003 : On the wave age dependence of wind stress over pure wind seas. J. Geophys. Res., 108, 8062, doi:10.1029/2000JC000715.

Hara, T., and S. E. Belcher, 2002: Wind forcing in the equilibrium range of wind-wave spectra. J. Fluid Mech., 470, 223-245.

$\longrightarrow$, and —-, 2004: Wind profile and drag coefficient over mature ocean surface wave spectra. J. Phys. Oceanogr., 34, 2345-2358.

Hwang, P. A., 2005: Temporal and spatial variation of the drag coefficient of a developing sea under steady wind-forcing. $J$. Geophys. Res., 110, C07024, doi:10.1029/2005JC002912.

Jähne, B., and K. S. Riemer, 1990: Two-dimensional wave number spectra of small-scale water surface waves. J. Geophys. Res., 95, 11 531-11 546.

_ , and H. Haußecker, 1998: Air-water gas exchange. Annu. Rev. Fluid Mech., 30, 443-468.

Janssen, P. A. E. M., 1989: Wave-induced stress and the drag of air flow over sea waves. J. Phys. Oceanogr., 19, 745-754.

Jarosz, E., D. A. Mitchell, D. W. Wang, and W. J. Teague, 2007: Bottom-up determination of air-sea momentum exchange under a major tropical cyclone. Science, 315, 1707-1709, doi:10.1126/science.1136466.

Jenkins, A. D., 1992: A quasi-linear eddy-viscosity model for the flux of energy and momentum to wind waves using conservation-law equations in a curvilinear coordinate system. $J$. Phys. Oceanogr., 22, 843-858.

- 1993: A simplified quasi-linear model for wave generation and air-sea momentum flux. J. Phys. Oceanogr., 23, 20012018.
_ 2001: Do strong winds blow waves flat? Wave Measurement and Analysis, B. Edge and J. Hemsley, Eds., ASCE Publication, 494-500.

Jones, I. S. F., and Y. Toba, Eds., 2001: Wind Stress over the Ocean. 1st ed. Cambridge University Press, 307 pp.

Komen, G. J., L. Cavaleri, M. Donelan, K. Hasselmann, S. Hasselmann, and P. A. E. M. Janssen, Eds., 1996: Dynamics and Modelling of Ocean Waves. 1st ed. Cambridge University Press, $532 \mathrm{pp}$.

Kudryavtsev, V. N., 2006: On the effect of sea drops on the atmospheric boundary layer. J. Geophys. Res., 111, C07020, doi:10.1029/2005JC002970.

_, and V. K. Makin, 2001: The impact of air-flow separation on the drag of the sea surface. Bound.-Layer Meteor., 98, 155171.

—, and _ 2007: Aerodynamic roughness of the sea surface at high winds. Bound.-Layer Meteor., 125, 289-303, doi:10.1007/s10546-007-9184-7.

- - _ - and B. Chapron, 1999: Coupled sea surfaceatmosphere model 2. Spectrum of short wind waves. J. Geophys. Res., 104, 7625-7640.

Kukulka, T., and H. Hara, 2008: The effect of breaking waves on a coupled model of wind and ocean surface waves. Part I: Mature seas. J. Phys. Oceanogr., 38, 2145-2163.

,$- \ldots$, and S. Belcher, 2007: A model of the air-sea momentum flux and breaking-wave distribution for strongly forced wind waves. J. Phys. Oceanogr., 37, 1811-1828.

Makin, V. K., 2005: A note on the drag of the sea surface at hurricane winds. Bound.-Layer Meteor., 115, 169-176.

- and V. N. Kudryavtsev, 2002: Impact of dominant waves on sea drag. Bound.-Layer Meteor., 103, 83-99.

Melville, W. K., 1996: The role of surface-wave breaking in air-sea interaction. Annu. Rev. Fluid Mech., 28, 279-321.

_ , and P. Matusov, 2002: Distribution of breaking waves at the ocean surface. Nature, 417, 58-63, doi:10.1038/417058a.

Phillips, O., 1958: The equilibrium range in the spectrum of windgenerated waves. J. Fluid Mech., 4, 426-434. , 1977: The Dynamics of the Upper Ocean. 2nd ed. Cambridge University Press, $336 \mathrm{pp}$.

, 1985: Spectral and statistical properties of the equilibrium range in wind-generated gravity waves. J. Fluid Mech., 156, 505-531.

Powell, M. D., P. J. Vickery, and T. A. Reinhold, 2003: Reduced drag coefficient for high wind speeds in tropical cyclones. Nature, 422, 279-283, doi:10.1038/nature01481.

Reul, N., H. Branger, and J.-P. Giovanangeli, 1999: Air flow separation over unsteady breaking waves. Phys. Fluids, 11, 19591961.

Smedman, A., X. G. Larsén, U. Högström, K. K. Kahma, and H. Pettersson, 2003: Effect of sea state on the momentum exchange over the sea during neutral conditions. J. Geophys. Res., 108, 3367, doi:10.1029/2002JC001526.

Thorpe, S., 2005: The Turbulent Ocean. 1st ed. Cambridge University Press, 439 pp.

Toba, Y., and N. Ebuchi, 1991: Sea-surface roughness length fluctuating in concert with wind and waves. J. Oceanogr. Soc. Japan, 47, 63-79.

—, N. Iida, H. Kawamura, N. Ebuchi, and I. S. Jones, 1990: Wave dependence of sea-surface wind stress. J. Phys. Oceanogr., 20, 705-721.

Tolman, H. L., 1999: User manual and system documentation of WAVEWATCH-III, version 1.18 , NOAA/NWS/NCEP/ OMB Tech. Note 166, 139 pp. 